\title{
A Prognostic Model Based on Six Metabolism-Related Genes in Colorectal Cancer
}

\author{
Yuan-Lin Sun, Yang Zhang, Yu-Chen Guo, Zi-Hao Yang, and Yue-Chao Xu \\ Department of Gastrointestinal Surgery, The First Hospital, Jilin University, Changchun, 130021 Jilin Province, China \\ Correspondence should be addressed to Yue-Chao Xu; ycxu@jlu.edu.cn
}

Received 21 April 2020; Revised 22 July 2020; Accepted 4 August 2020; Published 1 September 2020

Academic Editor: Serena Rinaldo

Copyright (C) 2020 Yuan-Lin Sun et al. This is an open access article distributed under the Creative Commons Attribution License, which permits unrestricted use, distribution, and reproduction in any medium, provided the original work is properly cited.

\begin{abstract}
An increasing number of studies have shown that abnormal metabolism processes are closely correlated with the genesis and progression of colorectal cancer (CRC). In this study, we systematically explored the prognostic value of metabolism-related genes (MRGs) for CRC patients. A total of 289 differentially expressed MRGs were screened based on The Cancer Genome Atlas (TCGA) and the Molecular Signatures Database (MSigDB), and 72 differentially expressed transcription factors (TFs) were obtained from TCGA and the Cistrome Project database. The clinical samples obtained from TCGA were randomly divided at a ratio of $7: 3$ to obtain the training group $(n=306)$ and the test group $(n=128)$. After univariate and multivariate Cox regression analyses, we constructed a prognostic model based on 6 MRGs (AOC2, ENPP2, ADA, GPD1L, ACADL, and CPT2). KaplanMeier survival analysis of the training group, validation group, and overall samples proved that the model had statistical significance in predicting the outcomes of patients. Independent prognosis analysis suggested that this risk score might serve as an independent prognosis factor for CRC patients. Moreover, we combined the prognostic model and the clinical characteristics in a nomogram to predict the overall survival of CRC patients. Furthermore, gene set enrichment analysis (GSEA) was conducted to identify the enriched Kyoto Encyclopedia of Genes and Genomes (KEGG) pathways in the high- and low-risk groups, which might provide novel therapeutic targets for CRC patients. We discovered through the protein-protein interaction (PPI) network and TF-MRG regulatory network that 7 hub genes were retrieved from the PPI network and 4 kinds of differentially expressed TFs (NR3C1, MYH11, MAF, and CBX7) positively regulated 4 prognosis-associated MRGs (GSTM5, PTGIS, ENPP2, and P4HA3).
\end{abstract}

\section{Introductions}

Statistically, CRC is the fourth leading malignancy worldwide regarding its incidence, occupying about $10.2 \%$ of the total tumor incidence [1]. Moreover, nearly a half of patients die within 5 years after they are diagnosed [2]. Most CRC cases progress from polypoid adenomas to high-grade dysplasia, then to adenoma-adenocarcinoma [3], and this process usually takes over 10 years $[3,4]$. Currently, although various treatments have been developed for CRC, the patients' prognosis still remains unsatisfactory, especially for patients with lymph node metastasis [5]. For the time being, the TNM classification system is the major pathological staging method, which can hardly accurately evaluate the prognosis of CRC. With the progress of the genome-sequencing technologies and the protein function research, an increasing number of studies about the biomarkers to predict the development and prognosis of tumor have emerged. Microsatellite Instability (MSI) status and TP53 mutation status are associated with the event-free survival after neoadjuvant chemotherapy [6]. And the high expression of PIWIinteracting RNA (piRNA) predicts poor prognosis of colorectal cancer [7]. However, the clinical application of biomarkers is still in development.

The role of metabolic disorder in the development and therapy of malignant tumors remains a research hotspot. In the mouse model, the high cholesterol levels associate with the enhanced phosphorylation of Akt, accelerating breast cancer cell growth in experiment in vitro [8]. Shu et al. believed that multiple glyceryl phosphatides, especially phosphatidylcholine and phosphatidylethanolamine, 


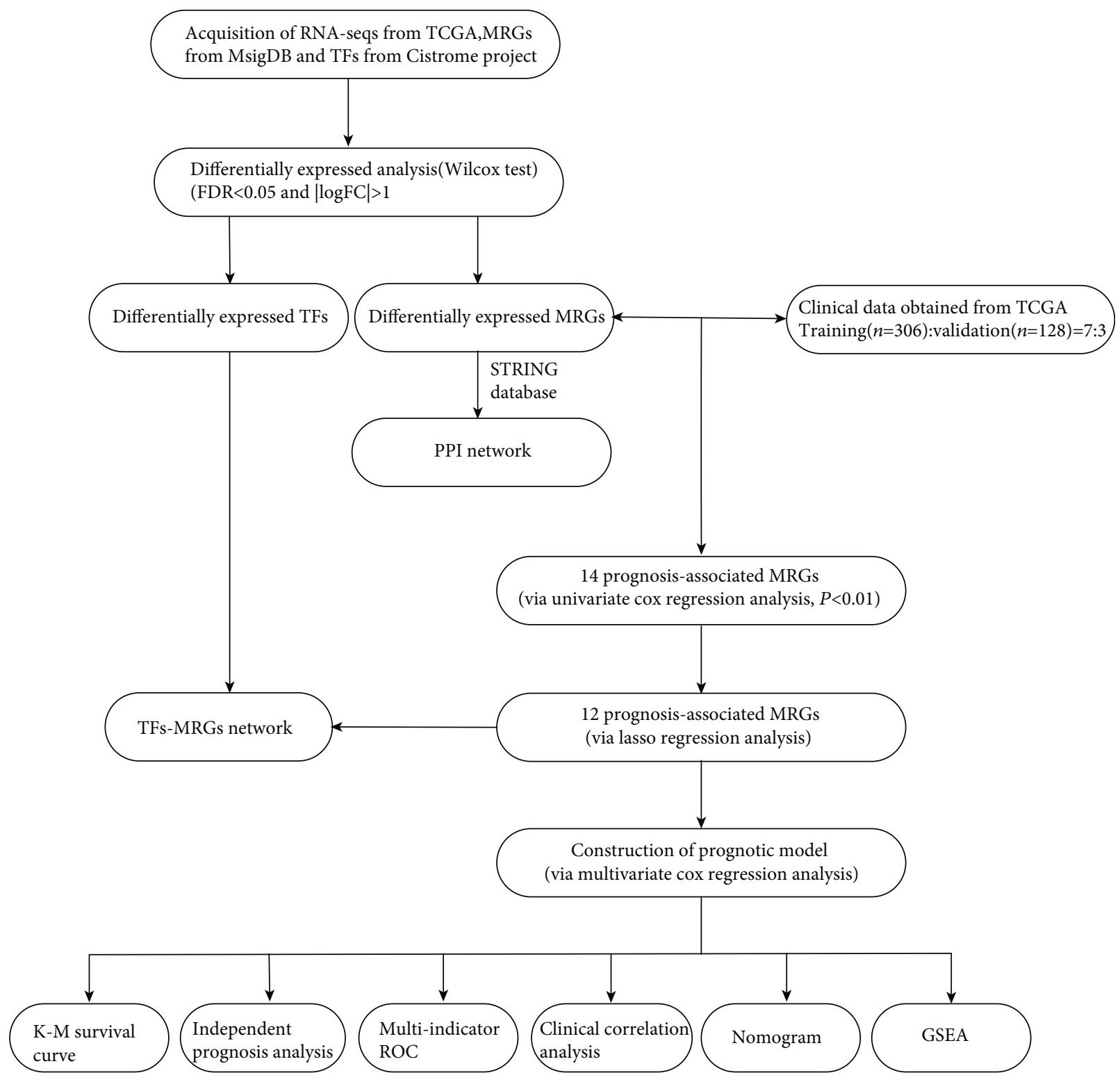

FIgUre 1: The flow diagram of the study.

were negatively correlated with the risk of CRC [9]. To inhibit the glycolytic pathway of tumor, shikonin could suppress the activity of PKM2 [10]. Therefore, the therapy strategy targeting to the metabolism might provide novel therapeutic promise for CRC patients.

In this study, we constructed a MRG-based prognostic model to systematically evaluate the prognosis of CRC patients. Kaplan-Meier survival analysis and independent prognosis analysis demonstrated the prognostic value of the prognostic model. Next, hierarchical analysis for high- and low-risk groups in CRC patients with GSEA might provide the novel therapeutic targets for CRC patients. Moreover, the PPI network and TFMRG network offered more reference for understanding the molecular relationship and molecular regulatory mechanisms.

\section{Materials and Methods}

2.1. Data Collection. The Cancer Genome Atlas (TCGA) data portal (https://portal.gdc.cancer.gov/) was used to acquire RNA sequences extracted from 482 tumor samples and 41 normal or paratumor samples and associated clinical data. The MSigDB v7.0 (c2: curated gene sets: KEGG gene sets, gene symbols) was used to obtain the MRGs. The TFs were obtained through the Cistrome Project database (http://www.cistrome.org/) [11].

2.2. Further Extraction of the MRG Data. The MRGs were obtained through the KEGG gene sets in MSigDB and intersected with all genes obtained in TCGA. The Wilcoxon test was utilized for differential analysis to obtain the differentially expressed MRGs according to the thresholds of false discovery rate $(\mathrm{FDR})<0.05$ and $\mid \log _{2}$ fold change $\mid(|\log \mathrm{FC}|)>1$. 


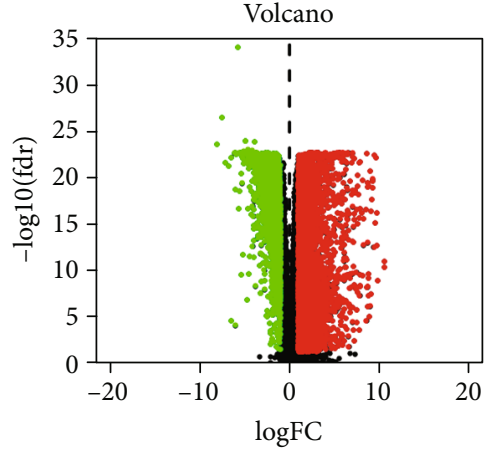

(a)

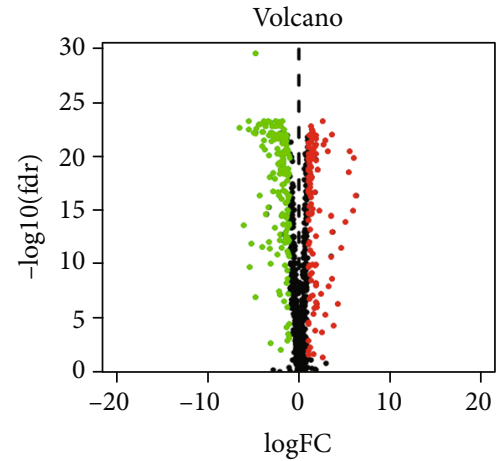

(b)

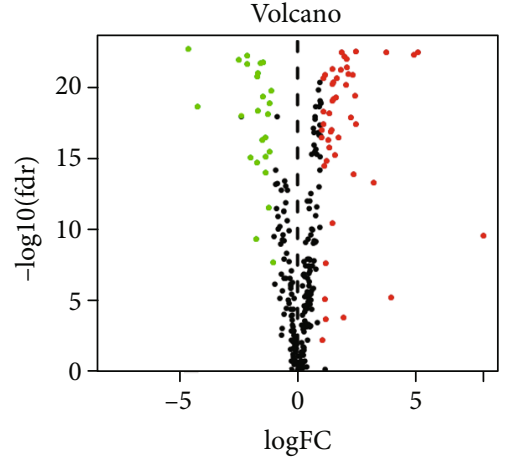

(c)
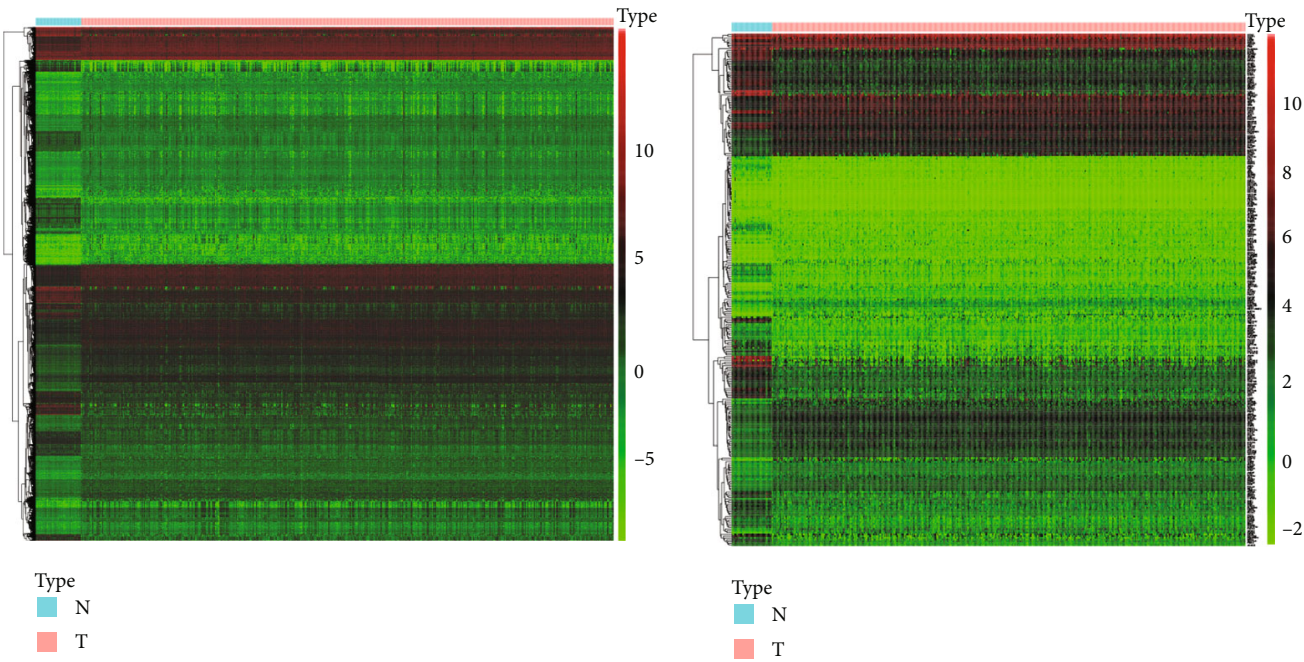

(d)

(e)

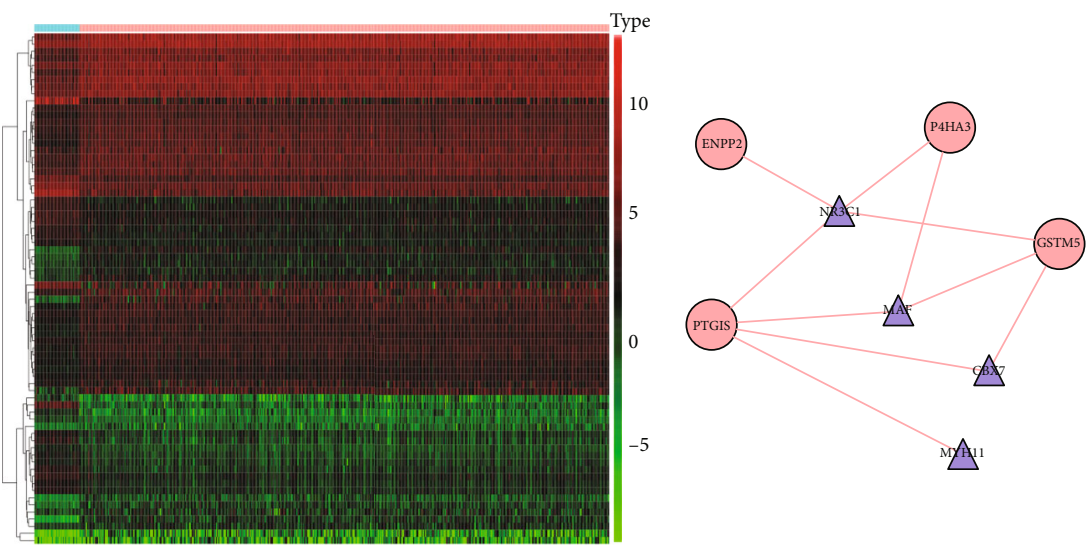

Type
$\mathrm{N}$
$\mathrm{T}$

(f)

(g)

FIGURE 2: The expression of differentially expressed genes (DEGs), differentially expressed MRGs, and differentially expressed TFs. (a-f) The heat maps and volcano plots of differentially expressed genes (DEGs), differentially expressed MRGs, and differentially expressed TFs. (g) shows the TF-MRG regulatory network, in which the red line indicates a positive regulation, the triangles represent TFs, and the red ellipses represent high-risk prognosis-associated MRGs. We found that the TFs positively regulated the prognosis-associated MRGs.

2.3. The Construction of the Protein-Protein Interaction (PPI) Network. In order to explore the underlying mechanisms of the interactions among the MRGs, we constructed a PPI network related to the differentially expressed MRGs with the
Search Tool for the Retrieval of Interacting Genes (STRING) 11.0 (https://string-db.org). Meanwhile, Molecular Complex Detection (MCODE), the tool of the Cytoscape 3.7.2 software [12], was utilized to retrieve the hub genes of PPI network. 


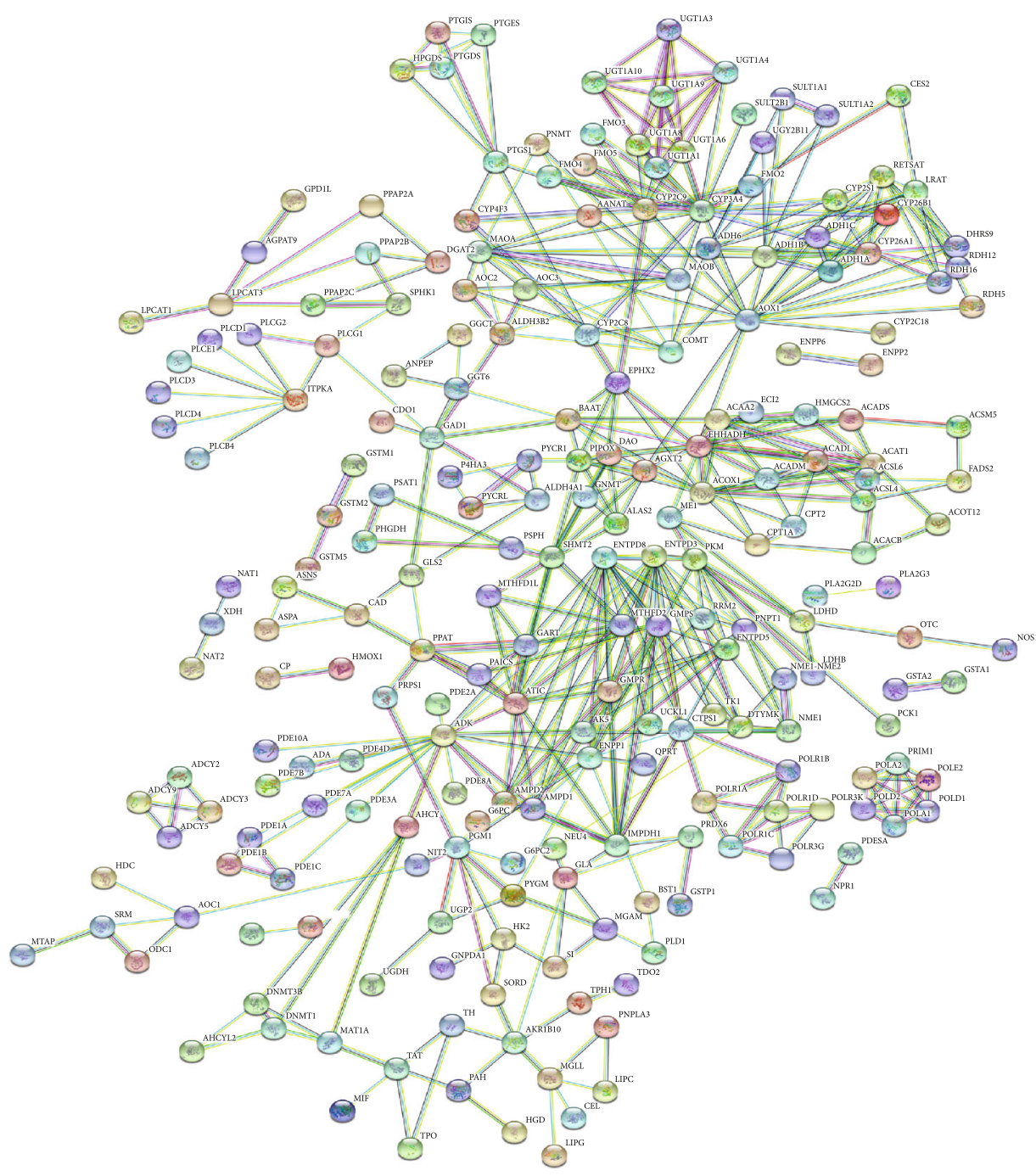

(a)

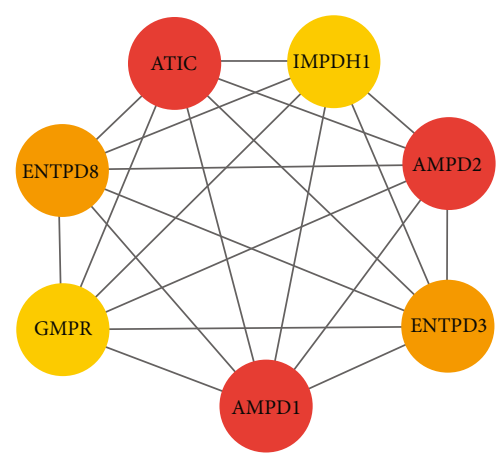

(b)

FIgURE 3: (a) The PPI network among differentially expressed MRGs. (b) The 7 hub genes of the PPI network.

2.4. The Preliminary Validation of the Prognosis-Associated MRGs. To guarantee accuracy and objectivity, patients with missing survival time data or with a survival time of fewer than 30 days were excluded, since these patients might have died from other acute fatal diseases (heart disease and cerebral infarction), rather than CRC. Afterward, we used the "caret package" in the R software to divide patients into a training group and a validation group at a ratio of $7: 3$, and the "survival package" in the R software was employed to conduct univariate Coxregression analysis to obtain the prognosisassociated MRGs that were highly correlated with survival $(P<0.01)$. To avoid overfitting when constructing the prognostic model, the training group was subject to lasso regression analysis [13] and partial likelihood deviance to screen prognosis-associated MRGs, among which, the prognosisassociated MRGs with hazard ratio (HR) $>1$ were defined as the high-risk MRGs and the prognosis-associated MRGs with hazard ratio $(\mathrm{HR})<1$ were defined as the low-risk MRGs.

2.5. The Construction of the TF-MRG Network. TFs were obtained from the Cistrome Project database, and the differ- entially expressed TFs were extracted based on the obtained differentially expressed genes (DEGs) according to the thresholds of FDR $<0.05$ and $|\operatorname{logFC}|>1$. The prognosisassociated MRGs and differentially expressed TFs were subjected to Pearson correlation analysis (Cor $>0.4$ and $P$ value $<0.001)$ to acquire the TFs and prognosis-associated MRGs used for the construction of the TF-MRG regulatory network, which was visualized with Cytoscape 3.7.2 software.

2.6. The Construction of the Prognostic Model. The "survival package" and "survimer package" in R software were employed for multivariate Cox regression analysis of the training group and validation group to obtain MRGs, among which we further uncovered their functional correlations in Gene Ontology (GO) and Kyoto Encyclopedia of Genes and Genomes (KEGG) through Database for Annotation, Visualization and Integrated Discovery (DAVID) and the risk score for the construction of the prognostic model. Patients in the training group and validation group were divided into high- and low-risk groups according to the median risk score. The survival function of $\mathrm{R}$ software was 
TABLE 1: The grouping of patients.

\begin{tabular}{|c|c|c|}
\hline Variables & $\begin{array}{l}\text { Training }(n=306) \\
\text { Cases (percentage) }\end{array}$ & $\begin{array}{l}\text { Validation }(n=128) \\
\text { Cases (percentage) }\end{array}$ \\
\hline \multicolumn{3}{|l|}{ Age } \\
\hline$\leq 60$ & $90(29.41 \%)$ & $48(37.50 \%)$ \\
\hline$>60$ & $216(70.59 \%)$ & $80(62.50 \%)$ \\
\hline \multicolumn{3}{|l|}{ Gender } \\
\hline Female & $127(41.50 \%)$ & $68(53.13 \%)$ \\
\hline Male & $179(58.50 \%)$ & $60(46.88 \%)$ \\
\hline \multicolumn{3}{|l|}{ Tumor stage } \\
\hline Stage I & $51(16.67 \%)$ & $26(20.31 \%)$ \\
\hline Stage II & $118(38.56 \%)$ & $41(32.03 \%)$ \\
\hline Stage III & $84(27.45 \%)$ & 35 (27.34\%) \\
\hline Stage IV & $41(13.40 \%)$ & 23 (17.97\%) \\
\hline NA & $12(3.92 \%)$ & $3(2.34 \%)$ \\
\hline \multicolumn{3}{|l|}{ T stage } \\
\hline $\mathrm{T} 1+\mathrm{Tis} / \mathrm{T} 1$ & $10(3.27 \%)$ & $3(2.34 \%)$ \\
\hline $\mathrm{T} 2$ & $53(17.32 \%)$ & 27 (21.09\%) \\
\hline $\mathrm{T} 3$ & $210(68.63 \%)$ & 87 (67.97\%) \\
\hline $\mathrm{T} 4$ & $33(10.78 \%)$ & $11(8.59 \%)$ \\
\hline \multicolumn{3}{|l|}{$\mathrm{N}$ stage } \\
\hline No & $178(58.17 \%)$ & $74(57.81 \%)$ \\
\hline N1 & $73(23.86 \%)$ & $34(26.56 \%)$ \\
\hline $\mathrm{N} 2$ & $44(14.83 \%)$ & $30(23.44 \%)$ \\
\hline $\mathrm{Nx}$ & $1(0.33 \%)$ & 1 \\
\hline \multicolumn{3}{|l|}{ M stage } \\
\hline M0 & $230(75.16 \%)$ & $96(75.00 \%)$ \\
\hline M1 & $40(13.07 \%)$ & $23(17.97 \%)$ \\
\hline $\mathrm{Mx}$ & $31(10.13 \%)$ & $8(6.25 \%)$ \\
\hline NA & $5(1.63 \%)$ & $1(0.78 \%)$ \\
\hline
\end{tabular}

utilized to conduct Kaplan-Meier survival analyses on the high-risk group and low-risk group in the training group, validation group, and overall samples. The risk score and survival status curves reflected the distribution of patient risk score in the high- and low-risk groups, as well as the relationship between the risk score and the survival status. Heat maps denoted the changes in the expression of various significant prognostic MRGs in the high- and low-risk groups. The clinical characteristics obtained from TCGA, including age, gender, tumor stage, pathological T stage, pathological $\mathrm{N}$ stage, and pathological $\mathrm{M}$ stage, were subjected to independent prognosis analysis combined with the risk score of the prognostic model, to verify whether the prognostic model might serve as an independent factor to predict patient prognosis. Furthermore, the "survival ROC" in R software was adopted to plot the multi-indicator ROC curves for the training group, validation group, and overall samples, and the values of the areas under the curve (AUCs) were observed to verify the feasibility and accuracy of our prognostic model and the clinical characteristics in predicting the patients' prognosis.

2.7. The Nomogram for the Prognostic Model and Clinical Characteristics. To more intuitively predict the patient sur- vival time, the "rms package" in R software was used to plot the nomogram with a combination of the prognostic model and clinical characteristics (age, sex, and tumor stage). Additionally, the calibration curve and C-index were utilized to verify the consistency and integral veracity of the nomogram. The ROC curves for 1-, 3-, and 5-year patient survival were also plotted to examine the feasibility of the nomogram in predicting the patient's survival rate in chronological order.

2.8. Gene Set Enrichment Analysis. To judge the potential relationship of each enriched KEGG pathway in the highand low-risk groups, the overall samples were subjected to GSEA [14]. The corresponding normalized enrichment scores (NES) in each KEGG enriched pathway were observed to judge whether this pathway was active in the high-risk group or the low-risk group. If NES $>0$, the pathway was active in the high-risk group; otherwise, if NES $<0$, the pathway was active in the low-risk group. The top 5 most active pathways in the high- and low-risk groups (FDR $<0.25$ indicated statistical significance) were selected for further visualization analysis.

\section{Results}

3.1. Data Acquisition. The main idea of the study is shown in Figure 1. We obtained RNA sequences from 481 tumor tissues and 41 paracarcinoma or nontumor tissues from TCGA-FPKM, which were then subjected to differential analysis using the "limma package" in R software. A total of 6475 DEGs (Table S1) were obtained according to the thresholds of $\mathrm{FDR}<0.05$ and $|\log \mathrm{FC}|>1$, including 4431 upregulated and 2044 downregulated ones (Figures 2(a) and 2(d)). Besides, the genes enriched in the metabolismrelated pathways were identified from the KEGG gene sets of MSigDB, which were deemed as MRGs. Altogether, 944 MRGs related to CRC were obtained after integrated analysis of the RNA sequences obtained from TCGAFPKM, which were subjected to differential analysis by the "limma package" in R software. In line with the thresholds of FDR $<0.05$ and $|\operatorname{logFC}|>1,289$ differentially expressed MRGs (Table S2) in total, including 163 downregulated and 126 upregulated ones, were obtained (Figures 2(b) and 2(e)). Meanwhile, 318 TFs were acquired from the Cistrome Project database, and then 72 differentially expressed TFs (Table S3) were obtained through integrated analysis of the DEGs based on the thresholds of FDR $<0.05$ and $|\log \mathrm{FC}|>1$, including 47 upregulated and 25 downregulated ones (Figures 2(c) and 2(f)).

3.2. The PPI Network. We aggregately screened out 487 interaction pairs composed of 227 differentially expressed MRGs to construct the PPI network (Figure 3(a)) with a confidence of 0.900 and filtration of isolated differentially expressed MRGs. And as shown in Figure 3(b), via MCODE of Cytoscape 3.7.2, 7 hub differentially expressed MRGs were selected from the PPI network.

3.3. The Acquisition of Prognostic-Associated MRGs. Patients conforming to the screening criteria were randomly divided at a ratio of $7: 3$ into a training group $(n=306)$ 


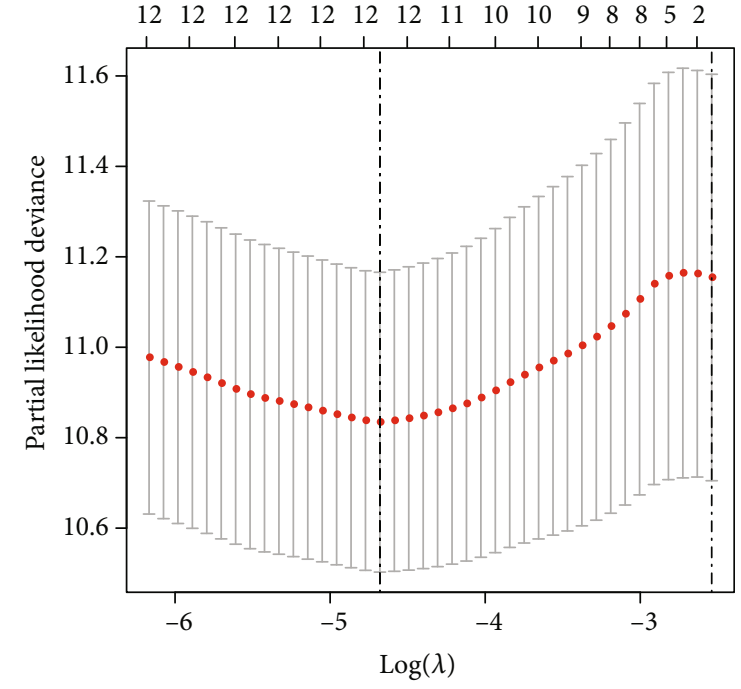

(a)

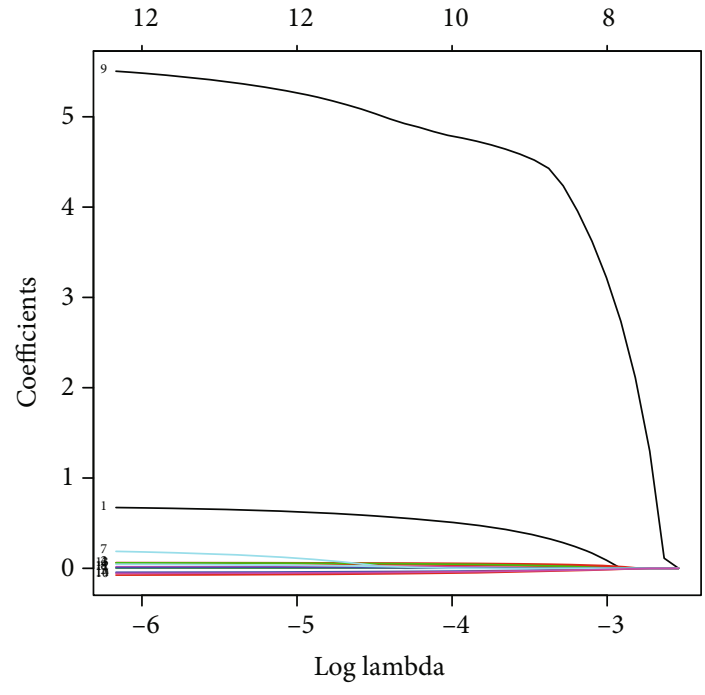

(b)

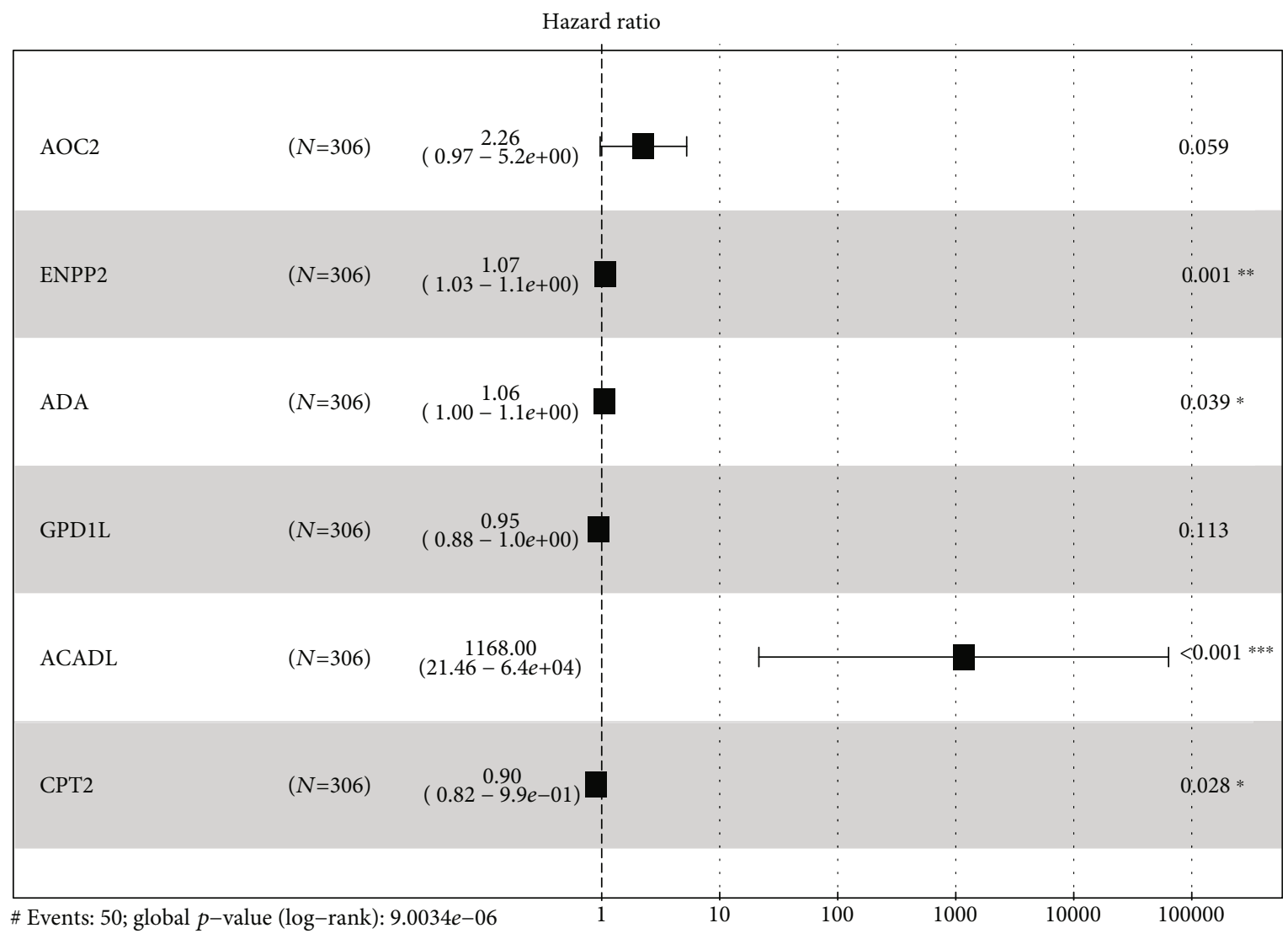

AIC: 469.94; concordance index: 0.7

(c)

FIGURE 4: The process of constructing MRG-based prognostic model. (a, b) Lasso regression analysis and partial likelihood deviance were applied on the identification of 12 prognosis-associated MRGs in the training group. (c) The six MRGs used for the construction of the prognostic model.

and validation group $(n=128)$ (Table 1$)$. On the basis of univariate Cox regression analysis combined with MRG expression quantities and patients' survival data, 14 MRGs significantly related to patients' survival were obtained $(P<0.01)$. Then, the 12 prognosis-associated MRGs were acquired through lasso regression analysis and partial likelihood deviance (Figures 4(a) and 4(b)). As is shown in Table 2, 9 high-risk prognosis-associated MRGs (ACADL, ENPP2, GPX3, PTGIS, ADH1B, P4HA3, ADA, AOC2, and GSTM5) $(\mathrm{HR}>1)$ and 3 low-risk prognosis- 
TABLE 2: The 12 prognosis-associated MRGs.

\begin{tabular}{lcccc}
\hline Genes & HR & HR.95L & HR.95H & $P$ value \\
\hline ACADL & 2763.714 & 54.38402 & 140447.7 & $7.69 E-05$ \\
ENPP2 & 1.071757 & 1.034298 & 1.110573 & 0.000135 \\
GPX3 & 1.015988 & 1.006999 & 1.025058 & 0.000469 \\
PTGIS & 1.142533 & 1.057611 & 1.234274 & 0.000721 \\
ADH1B & 1.106607 & 1.043144 & 1.17393 & 0.000775 \\
GPD1L & 0.901048 & 0.839121 & 0.967546 & 0.004129 \\
P4HA3 & 1.48786 & 1.133596 & 1.952835 & 0.004187 \\
PAFAH2 & 0.847885 & 0.755554 & 0.951499 & 0.005029 \\
CPT2 & 0.878993 & 0.80283 & 0.962382 & 0.005284 \\
ADA & 1.079064 & 1.021351 & 1.140038 & 0.006663 \\
AOC2 & 2.620745 & 1.297612 & 5.293035 & 0.007223 \\
GSTM5 & 3.900058 & 1.394631 & 10.90644 & 0.009489 \\
\hline
\end{tabular}

associated MRGs (GPD1L, PAFAH2, and CPT2) $(\mathrm{HR}<1)$ were screened out.

3.4. The Regulatory Network between Differentially Expressed TFs and Prognostic-Associated MRGs. We carried out Pearson correlation analysis (Cor $>0.4, \quad P<0.001)$ for 12 prognosis-associated MRGs and 72 DETFs and then screened 4 prognosis-associated MRGs (GSTM5, PTGIS, ENPP2, and P4HA3) and 4 differentially expressed TFs (NR3C1, MYH11, MAF, and CBX7) to construct the TFMRG regulatory network, which was visualized using the Cytoscape 3.7.2 software for intuitive observation. Obviously, the 4 differentially expressed TFs positively regulated the 4 prognosis-associated MRGs (Figure 2(g)).

3.5. The Six-MRG Prognostic Model. To construct the MRGbased prognostic model, we carried out multivariate Cox regression analysis on the 12 prognosis-associated MRGs. Finally, 6 MRGs (AOC2, ENPP2, ADA, ACADL, GPD1L, and CPT2), together with the corresponding coefficients, were obtained (Figure 4(c)). Eventually, the risk score was obtained, which was calculated as follows: risk score $=$ the expression of AOC2 $* 0.813994+\mathrm{ENPP} 2 * 0.065392+$

$\mathrm{ADA} * 0.060378+\mathrm{GPD} 1 \mathrm{~L} *(-0.05543)+\mathrm{ACADL} *$

$7.063052+\mathrm{CPT} 2 *(-0.10342)$. Using the median risk score of 0.774 , the patients with risk score $\geq 0.774$ were classified as the high-risk group, while those with the risk score $<0.774$ were classified as the low-risk group. Table 3 shows the results of GO and KEGG functional enrichment analyses for the MRGs of the prognostic model. GPD1L, AOC2, ACADL and CPT2, ACADL and AOC2, and ACADL were, respectively, active in GO terms, oxidation-reduction process, fatty acid beta-oxidation, and electron carrier activity included, with statistical significance $(P<0.05)$. CPT2 and ACADL were all enriched in KEGG pathways including fatty acid degradation, fatty acid metabolism, and PPAR signaling pathway with statistical significance $(P<0.05)$. Figures 5(a), $5(\mathrm{e})$, and 5(i) show survival curves based on the prognostic model in the training group, validation group, and overall samples. In the training group, the 5-year survival rate for
TABLE 3: GO and KEGG functional enrichment analysis for the MRGs of the prognostic model.

\begin{tabular}{lccc}
\hline Term & Count & Genes & $P$ value \\
\hline $\begin{array}{l}\text { GO:0055114 oxidation- } \\
\text { reduction process }\end{array}$ & 3 & $\begin{array}{c}\text { GPD1L, AOC2, } \\
\text { ACADL }\end{array}$ & 0.011559 \\
$\begin{array}{l}\text { GO:0006635 fatty } \\
\text { acid beta-oxidation }\end{array}$ & 2 & CPT2, ACADL & 0.013035 \\
$\begin{array}{l}\text { GO:0009055 electron } \\
\text { carrier activity }\end{array}$ & 2 & AOC2, ACADL & 0.026378 \\
$\begin{array}{l}\text { hsa00071: fatty acid } \\
\text { degradation }\end{array}$ & 2 & CPT2, ACADL & 0.030166 \\
$\begin{array}{l}\text { hsa01212: fatty acid } \\
\text { metabolism }\end{array}$ & 2 & CPT2, ACADL & 0.034415 \\
$\begin{array}{l}\text { hsa03320: PPAR } \\
\text { signaling pathway }\end{array}$ & 2 & CPT2, ACADL & 0.047773 \\
\hline
\end{tabular}

high-risk patients was about $47.9 \%$, while that for low-risk patients, it was about $75 \%$. In the validation group, the 5year survival rate for high-risk patients was lower than $40.7 \%$, and that in low-risk patients was about $60.9 \%$. The 5 -year survival rate for high-risk patients was about $46.7 \%$, and that for low-risk patients was about $77.9 \%$. The risk score plots (Figures 5(b), 5(f), and 5(j)) display the changes of risk score in high- and low-risk patients in training group, validation group, and overall samples, respectively. The survival status distribution graphs (Figures 5(c), 5(g), and 5(k)) show the changes of risk score and the patient survival distribution in the training group, validation group, and overall samples. Notably, the number of deaths increased and the survival time declined as the risk score increased in the training group, validation group, and overall samples. Heat maps (Figures 5(d), 5(h), and 5(l)) illustrate the variation trend of the expression of various prognosis-associated genes with the increase of the risk score. AOC2 showed the most obvious variation trend in the training group, validation group, and overall samples. Its expression quantity was positively correlated with the risk score, indicating that the expression quantity increased as the risk score increased. The expression of CPT2 and GPDL1 was negatively correlated with the risk score. To verify the influence of this prognostic model and various clinical characteristics on the patients' survival, we conducted an independent prognosis analysis. Univariate Cox regression (Table 4) and multivariate Cox regression analysis (Table 5) were performed on the clinical characteristics and risk score of the training group, validation group, and overall samples. The risk score might serve as an independent factor to predict the patient prognosis in the training group (Figures 6(a) and 6(b)), validation group (Figures 6(c) and 6(d)), and overall samples (Figures 6(e) and 6(f)) $(P<0.05)$. Additionally, the multi-indicator ROC curve was plotted, and the AUCs in the training group (Figure 6(g)), validation group (Figure 6(h)), and overall samples (Figure 6(i)) were $0.706,0.739$, and 0.716 , respectively, verifying that it was more feasible and accurate to use this model in predicting the outcomes of patients than the remaining clinical indicators. 


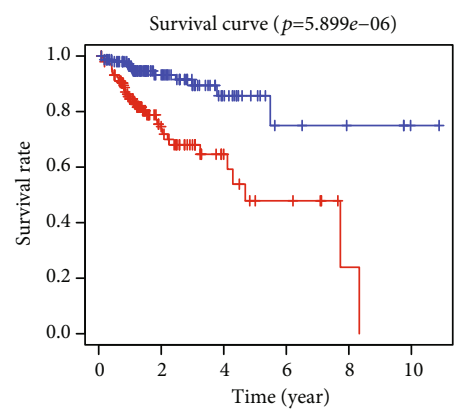

(a)

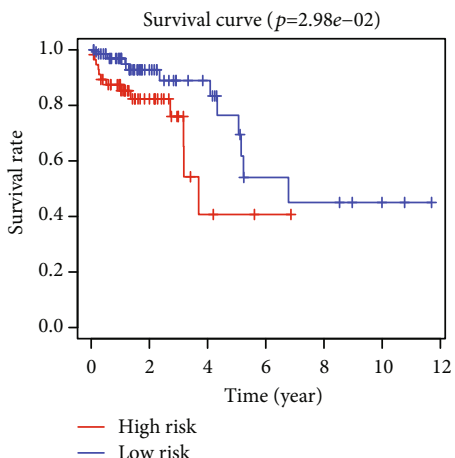

(e)

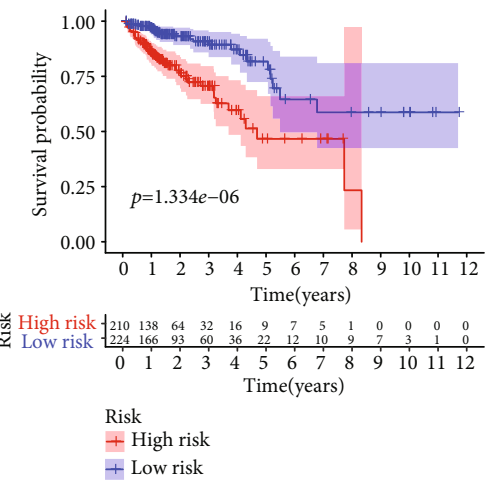

(i)

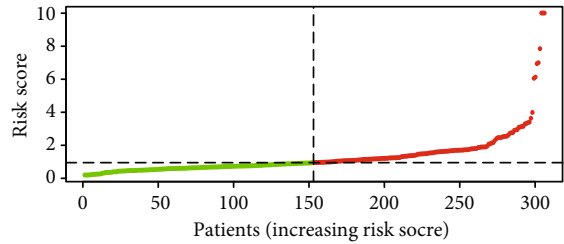

(b)

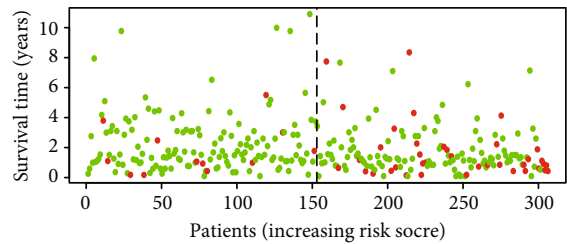

(c)

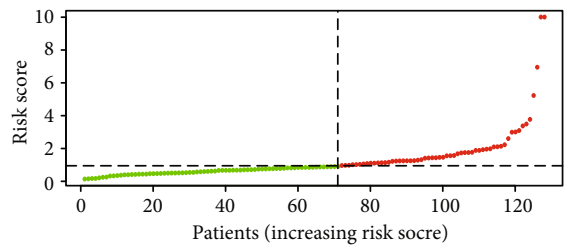

(f)

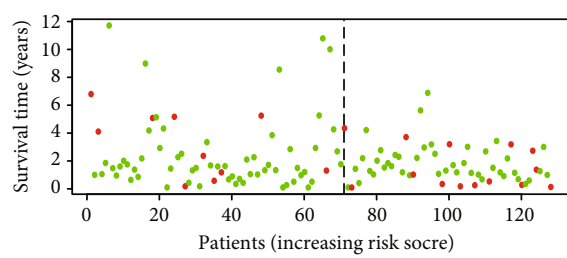

(g)

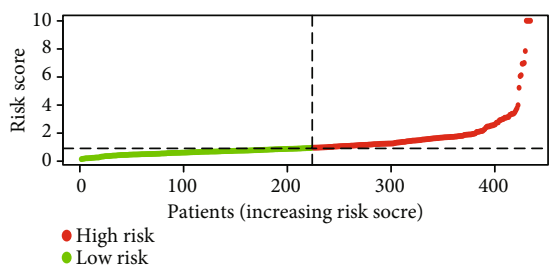

(j)

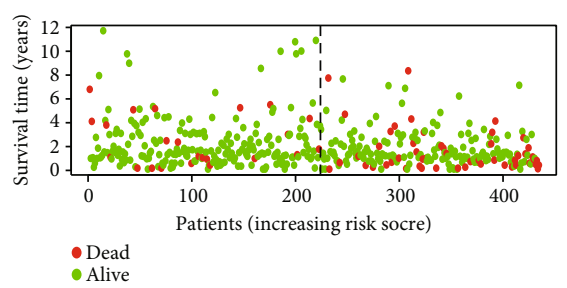

(k)

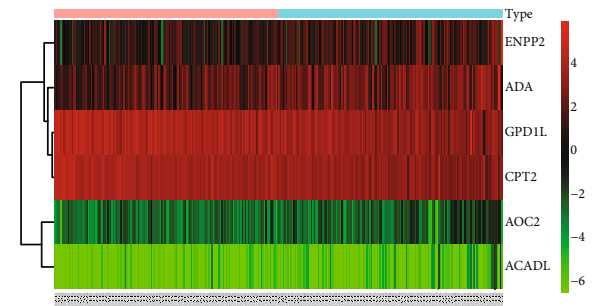

(d)

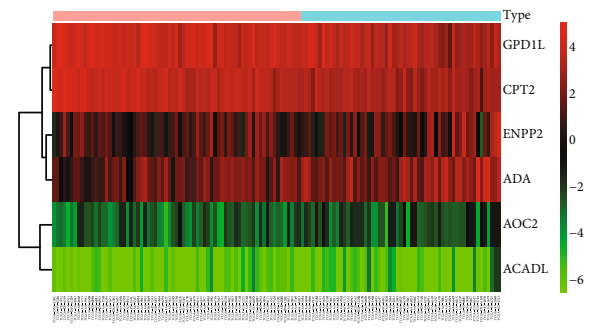

(h)

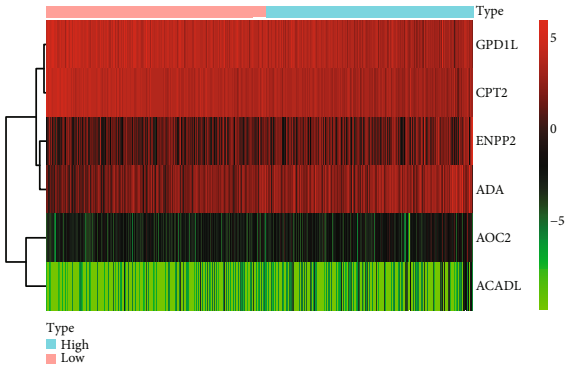

(l)

FIGURE 5: Kaplan-Meier survival analysis in training, validation, and overall samples. (a-l) Several showed the Kaplan-Meier survival curves, the risk score curves, the survival status plots, and heat maps in the training group, validation group, and overall samples.

3.6. The Clinical Correlation Analysis. Furthermore, we carried out clinical correlation analysis on various prognosisassociated MRGs and various clinical characteristics (age, gender, tumor stage, and pathological TNM system) so as to further explore the potential molecular regulatory relationships. $P<0.05$ was considered to denote statistical signif- icance, while $P<0.01$ suggested high statistical significance, and $P<0.001$ indicated significant statistical significance. To more intuitively present their relationships, we used the "ggpubr package" of R software to plot boxplots. Clearly, as shown in Figure 7(a), the ADA expression level was correlated with age, and patients older than 60 years had slightly 
TABLE 4: Univariate independent prognosis analysis.

\begin{tabular}{|c|c|c|c|c|c|c|}
\hline & \multicolumn{2}{|c|}{ Training group } & \multicolumn{2}{|c|}{ Validation group } & \multicolumn{2}{|c|}{ Overall samples } \\
\hline & Hazard ratio & $P$ & Hazard ratio & $P$ & Hazard ratio & $P$ \\
\hline$\overline{\text { Age }}$ & $1.021(0.993-1.050)$ & 0.147 & $1.064(1.014-1.117)$ & 0.011 & $1.030(1.005-1.054)$ & 0.016 \\
\hline Gender & $0.828(0.444-1.545)$ & 0.554 & $2.008(0.833-4.843)$ & 0.121 & $1.071(0.647-1.773)$ & 0.789 \\
\hline Stage & $3.100(2.127-4.519)$ & $<0.001$ & $2.375(1.442-3.911)$ & $<0.001$ & $2.857(2.114-3.861)$ & $<0.001$ \\
\hline $\mathrm{T}$ & $3.146(1.715-5.771)$ & $<0.001$ & $3.781(1.562-9.514)$ & 0.003 & $3.337(2.027-5.494)$ & $<0.001$ \\
\hline $\mathrm{N}$ & $2.210(1.549-3.154)$ & $<0.001$ & $2.662(1.549-4.574)$ & $<0.001$ & $2.371(1.764-3.186)$ & $<0.001$ \\
\hline $\mathrm{M}$ & $7.199(3.817-13.578)$ & $<0.001$ & $4.104(1.740-9.680)$ & 0.001 & $6.058(3.640-10.080)$ & $<0.001$ \\
\hline Risk score & $1.092(1.059-1.126)$ & $<0.001$ & $1.161(1.070-1.259)$ & $<0.001$ & $1.091(1.063-1.119)$ & $<0.001$ \\
\hline
\end{tabular}

TABLE 5: Multivariate independent prognosis analysis.

\begin{tabular}{|c|c|c|c|c|c|c|}
\hline & \multicolumn{2}{|c|}{ Training group } & \multicolumn{2}{|c|}{ Validation group } & \multicolumn{2}{|c|}{ Overall samples } \\
\hline & Hazard ratio & $P$ & Hazard ratio & $P$ & Hazard ratio & $P$ \\
\hline Age & $1.037(1.008-1.068)$ & 0.013 & $1.094(1.037-1.155)$ & $<0.001$ & $1.039(1.014-1.064)$ & 0.002 \\
\hline Gender & $0.625(0.321-1.218)$ & 0.167 & $2.089(0.814-5.356)$ & 0.125 & $0.904(0.537-1.522)$ & 0.704 \\
\hline Stage & $2.418(0.767-7.621)$ & 0.132 & $1.793(0.351-9.417)$ & 0.482 & $2.042(0.833-5.008)$ & 0.119 \\
\hline $\mathrm{T}$ & $1.532(0.789-2.978)$ & 0.208 & $2.171(0.740-6.639)$ & 0.158 & $1.744(0.986-3.084)$ & 0.056 \\
\hline $\mathrm{N}$ & $1.098(0.573-2.104)$ & 0.777 & $1.731(0.640-4.682)$ & 0.280 & $1.215(0.735-2.009)$ & 0.447 \\
\hline $\mathrm{M}$ & $1.445(0.287-7.288)$ & 0.655 & $0.773(0.091-6.537)$ & 0.813 & $1.308(0.384-4.459)$ & 0.667 \\
\hline Risk score & $1.056(1.021-1.092)$ & 0.002 & $1.178(1.071-1.295)$ & $<0.001$ & $1.059(1.030-1.089)$ & $<0.001$ \\
\hline
\end{tabular}

higher ADA expression level than those aged less than 60 years. As shown in Figure 7(b), the expression levels of CPT2 and GPDL1 were correlated with tumor stage, among which, the CPT2 expression level was significantly correlated with tumor stage. As the tumor stage advanced, the CPT2 expression level decreased accordingly, revealing a negative correlation. In tumor stage I and tumor stage II, the expression level of GPDL1 was consistent, but it decreased with the increase in tumor stage. As shown in Figure 7(c), the CPT2 expression level was considered to be significantly correlated with the pathological $\mathrm{N}$ stage. Patients with an advanced pathological $\mathrm{N}$ stage had lower expression than those with a lower pathological $\mathrm{N}$ stage, and the GPDL1 expression level displayed the same trend, which was related to the pathological $\mathrm{N}$ stage. The expression of ACADL and AOC2 showed significant correlations with pathological $\mathrm{N}$ stage, but their expression levels were low, with insignificant variation trends. For the pathological M stage (Figure $7(\mathrm{~d})$ ), the CPT2 expression level in patients with an advanced pathological M stage was lower. GPDL1 expression exhibited a similar trend, and the difference was statistically significant. The expression of the MRGs showed no obvious statistical significance with gender or pathological $\mathrm{T}$ stage.

3.7. The Four-Signature Nomogram. We integrated the prognostic model and clinical characteristics to predict patients' survival with a nomogram (Figure 8(a)). The age, gender, tumor stage, and risk score of the prognostic model were used as the elements for rating various risk factors of the patients, and the scores were added to obtain the total score, thus obtaining the corresponding predicted survival rate. Meanwhile, the 1-year, 3-year, and 5-year survival calibration curves (Figures 8(b)-8(d)) and C-index (0.806) indicated an ideal fitting and excellent accuracy of the nomogram. In the ROC curves (Figure 8(e)), the AUCs of the 1-, 3-, and 5year survival rates were $0.717,0.715$, and 0.740 , respectively, suggesting that this model relatively accurately predicted the survival rate for over $70 \%$ of the patients.

3.8. Gene Set Enrichment Analysis. To further explore the biological functions of the MRGs, we carried out GSEA on high- and low-risk groups, finding that 83 KEGG enriched pathways were active in the high-risk group, while 95 were active in the low-risk group. In CRC, various metabolismrelated pathways were mainly enriched in the low-risk group ( $n=38$ in total, including 18 with statistical significance at FDR $<0.25$, while they were rarely enriched in the highrisk group). Furthermore, 89 statistically significant KEGG enrichment pathways (FDR $<0.25$ ) were screened, among which, 49 were active in the high-risk group, while 40 were active in the low-risk group. The top 5 pathways with the highest NES in the high- and low-risk groups (Table 6) were selected for visualization analysis (Figure 9), intuitively demonstrating that the expression of genes enriched in KEGG pathways active in the high-risk group located above the $x$ axis were apparently higher than those in the low-risk group. Genes enriched in KEGG pathways that were active in the low-risk group and located below the $x$-axis also exhibited a similar trend.

\section{Discussion}

In total, a 6-MRG- (AOC2, ENPP2, ADA, GPD1L, ACADL, and CPT2) based prognostic model was constructed based on 


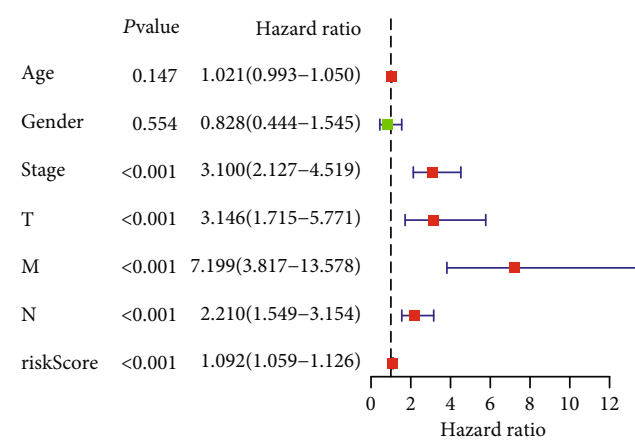

(a)

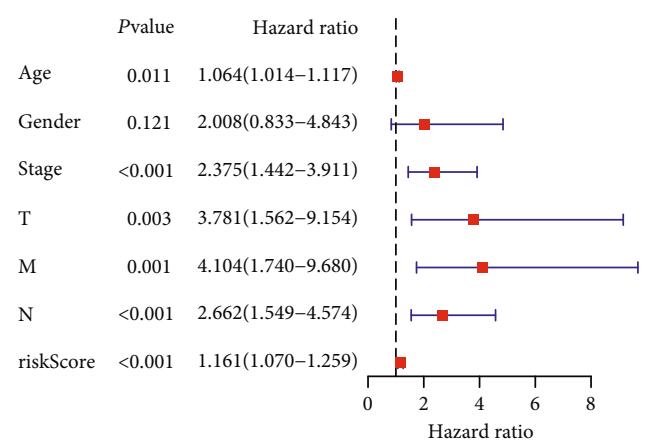

(c)

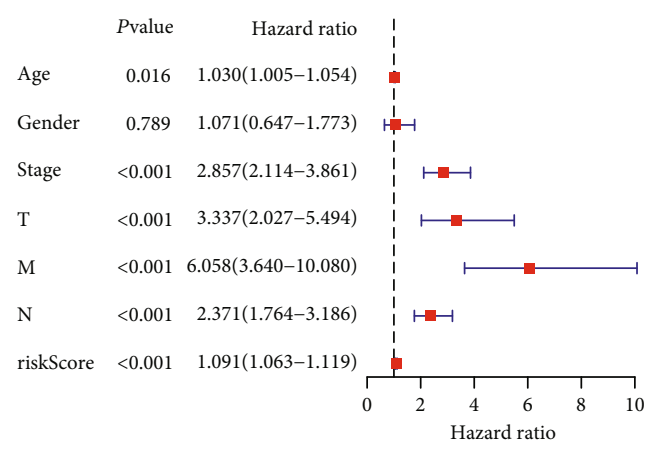

(e)

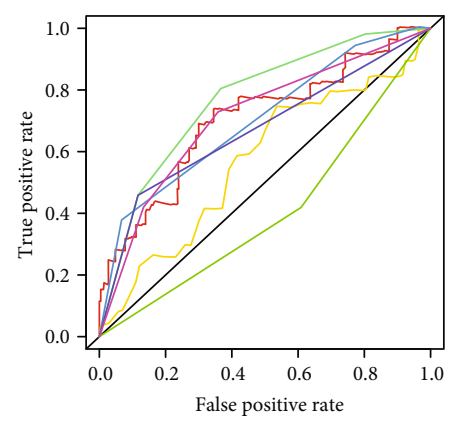

$$
\begin{aligned}
& \text { - Risk score }(\mathrm{AUC}=0.706) \\
& \text { - Age }(\mathrm{AUC}=0.570) \\
& \text { - Gender }(\mathrm{AUC}=0.405) \\
& \text { - Stage }(\mathrm{AUC}=0.769) \\
& \text { - } \mathrm{A}(\mathrm{AUC}=0.702) \\
& \text { - } \mathrm{M}(\mathrm{AUC}=0.671) \\
& \text { - } \mathrm{N}(\mathrm{AUC}=0.712)
\end{aligned}
$$

(g)

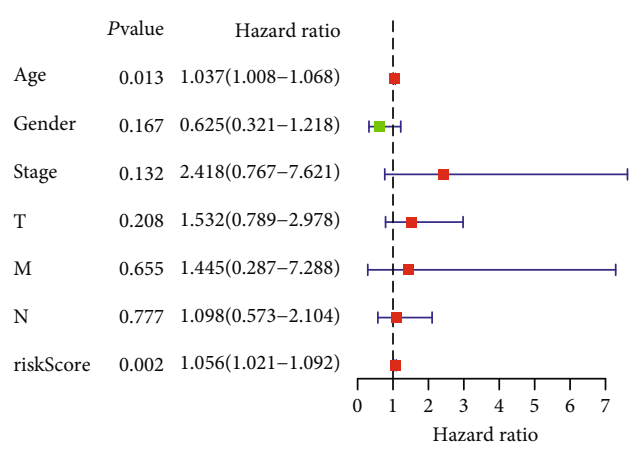

(b)

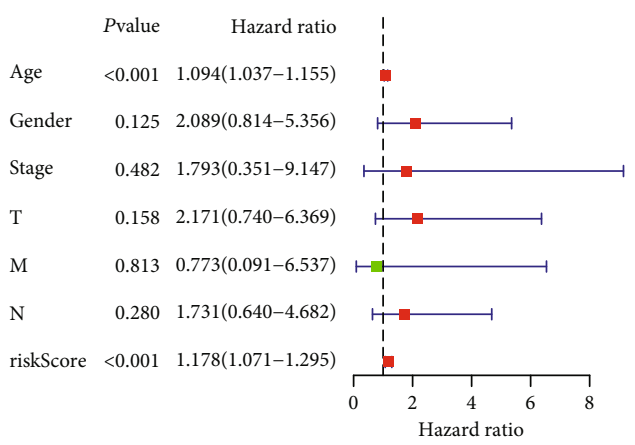

(d)

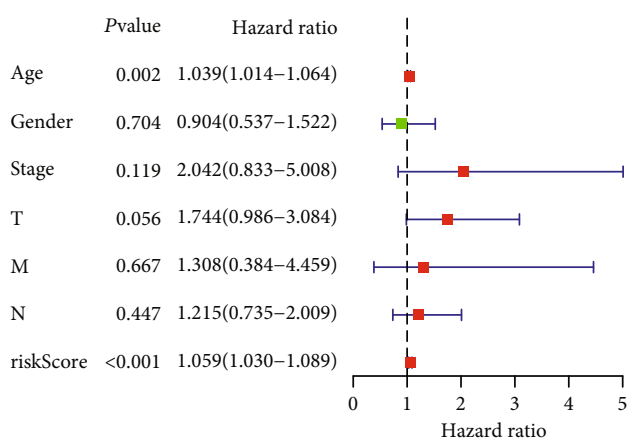

(f)

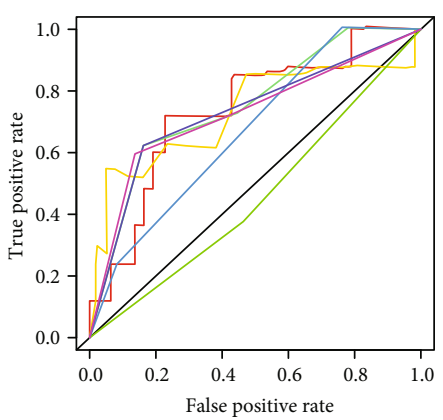

$$
\begin{aligned}
& \text { - Risk score }(\mathrm{AUC}=0.739) \\
& \text { - } \text { Age }(\mathrm{AUC}=0.730) \\
& \text { - Gender }(\mathrm{AUC}=0.456) \\
& \text { - Stage }(\mathrm{AUC}=0.752) \\
& \text { - } \mathrm{T}(\mathrm{AUC}=0.672) \\
& \text { - } \mathrm{M}(\mathrm{AUC}=0.730) \\
& \text { N }(\mathrm{AUC}=0.726)
\end{aligned}
$$

(h)

FIgURE 6: Continued. 


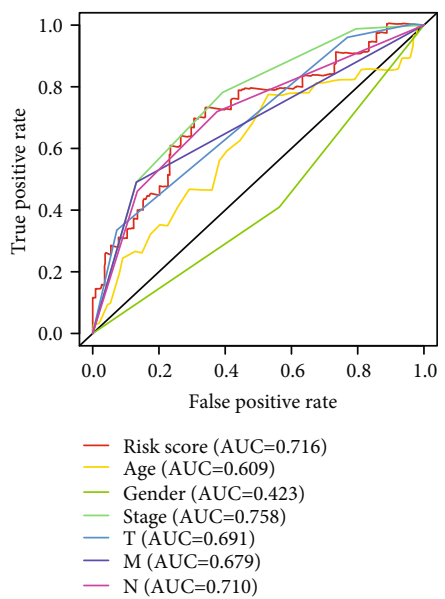

(i)

FIGURE 6: Independent prognosis analysis in training, validation, and overall samples. (a-f) Respectively showed univariate and multivariate independent prognosis analyses between clinical characteristics and risk score in the training group, validation group, and overall samples, where the red squares represent the high-risk signature and the green squares represent the low-risk signature. (g-i) The comparison of AUCs between clinical characteristics and risk score in the training group, validation group, and overall samples.

the CRC patient clinical characteristics and expression quantity of MRGs. The exploration on AOC2 is relatively limited, and the AOC2-like enzyme activity is detected in eye tissues [15]. ENPP2 is a gene that encodes autotaxin, which has been verified to be related to the growth and metastasis of melanoma tumor and stage I nonsmall cell lung cancer (NSCLC) $[16,17]$. And Zhao et al. revealed that autotaxin protein encoded by ENPP2 catalyzes the production of LPC into lysophosphatidic acid (LPA), and such lipid molecular metabolic reaction may be associated with the genesis and development of CRC [18]. Some studies indicated that ADA, participating in encoding an enzyme involved in purine metabolism, is downregulated in lymphocytes of advanced stage lung cancer [19]. Kelly et al. suggested that GPDL1 negatively regulated HIF- $1 \alpha$ protein expression in tumor cells, while suppressing miR-210 induced the high expression of GPDL1, which might become a new target in tumor treatment [20]. ACADL can encode an enzyme that participates in fatty acid and branched chain amino-acid metabolism. Hill et al. discovered that ACADL methylation might associate with the poor prognosis for breast cancer [21]. Regarding research on CPT2, Fujiwara et al. discovered that, in obesityand nonalcoholic steatohepatitis-driven hepatocellular carcinoma, the downregulation of CPT2 accelerated tumor progression [22]. In clinical correlation analysis, the CPT2 expression quantities were significantly correlated with stage and pathological $\mathrm{N}$ stage, and its expression quantities gradually decreased as the stage and pathological $\mathrm{N}$ stage advanced, thus possibly meaning the reduced expression quantities of CPT2 in advanced CRC. Meanwhile, GO functional annotation suggested that GPD1L, AOC2, ACADL and $\mathrm{CPT} 2$, ACADL and AOC2, and ACADL were respectively active in oxidation-reduction process, fatty acid betaoxidation, and electron carrier activity. Oxidation-reduction process was linked to the prognosis of hepatocellular carcinoma [23] and clear cell renal cell carcinoma [24]. The critical role of fatty acid beta-oxidation was also proven in the progression of cancer. It has been revealed that fatty acid beta-oxidation promotes proliferation of lymphatic endothelial cells by providing acetyl-CoA and regulates the differentiation of lymphatic endothelial cells with the epigenetic control of CPT1 [25]. And Wang et al. elucidated that JAK/STAT3-dependent fatty acid beta-oxidation is associated with breast cancer chemoresistance [26]. Xu et al. revealed that DNA methylation-driven genes in prostate adenocarcinoma were active in electron carrier activity [27]. What is more, KEGG pathway enrichment analysis uncovered that CPT2 and ACADL were both enriched in fatty acid degradation, fatty acid metabolism, and PPAR signaling pathway, which were all directly or indirectly involved in the process of lipid metabolism related to the progression of malignant tumors [28-31]. No doubt that the functional relationship among MRGs of the prognostic model provided compelling evidence for the role of metabolism in the progression of cancer from the molecular level. Upon the prognostic model constructed, Kaplan-Meier survival analysis for patients classified into high- and low-risk groups according to the median risk score in the training and validation groups verified the prognostic value of the prognostic model. The independent prognosis analysis validated that the risk score acquired had favorable statistical significance in predicting the patient prognostic outcomes. Additionally, the nomogram showed favorable accuracy in predicting the 1-, 3-, and 5-year survival rates of patients, contributing to systemically planning patient's follow-up.

We further explored the underlying multiple molecular relationships based on differentially expressed MRGs, among which, PPI network was constructed. From the PPI network, we identified 7 hub genes, namely, ATIC, IMPDH1, ENTPD8, AMPD2, GMPR, ENTPD3, and AMPD1. Ruan et al. found that the high expression quantity of IMPDH1 was related to the poor prognosis of malignant tumors, and the interaction between IMPDH1 and YB-1 was associated to the tumor metastasis, which might be a novel therapeutic 

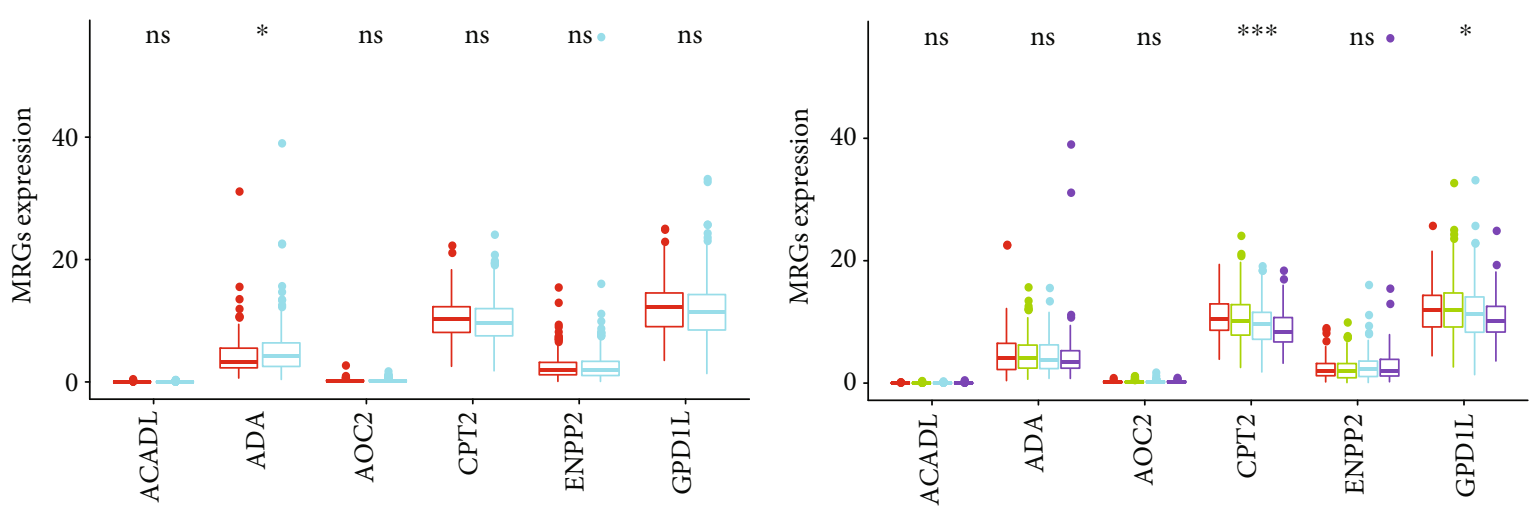

Type

官 M0

Type

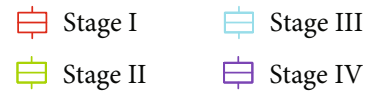

(a)

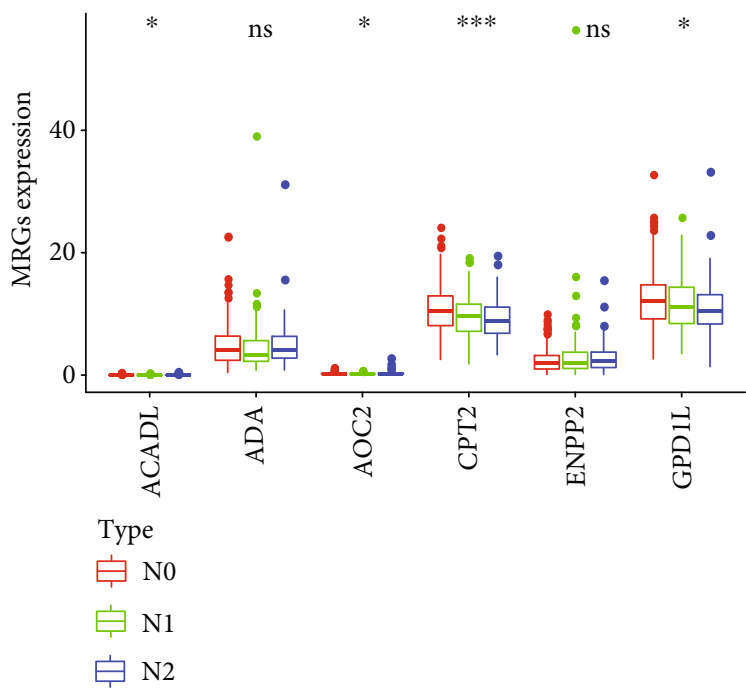

(b)

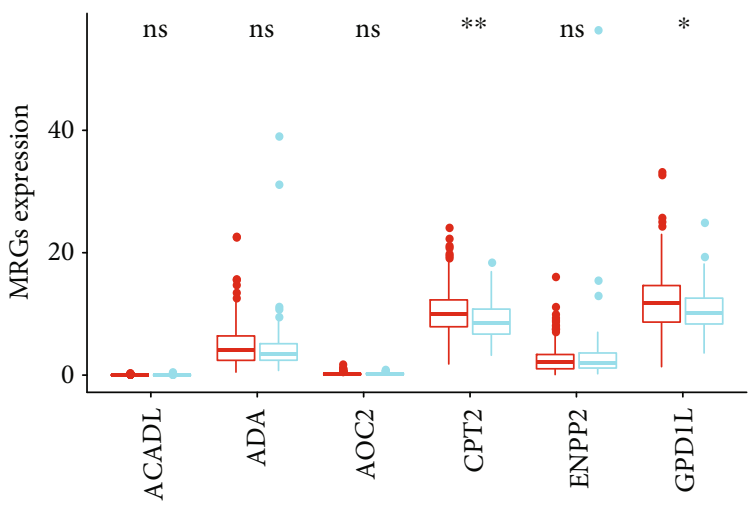

(c)

(d)

Figure 7: The clinical correlation analysis. $(\mathrm{a}-\mathrm{d})$ The boxplots showed the correlation between the expression level of the MRGs and the clinical characteristics, in which ${ }^{*} P<0.05,{ }^{* *} P<0.01$, and ${ }^{* * *} P<0.001$.

target [32]. An et al. revealed that ENTPD8, the related gene of metabolite cytidine, was low expressed in pancreatic cancer [33]. And AMPD2 was identified as a potential biomarker for predicting the poor prognosis of undifferentiated pleomorphic sarcoma functional genomics identifies [34]. GMPR was found that it could downregulate GTP-bound RhoGTPases and inhibited the further development of melanoma [35]. Feldbrugge et al. elucidated that the enzyme expressed by ENTPD3 prevented colon against inflammation and purinergic signaling regulated by ENTPD3 dominated neuroimmune interactions related to Crohn's disease [36]. AMPD1 could be regarded as the biomarker to predict the survival of breast cancer [37]. There is no doubt that our studies provided theoretical support for the interaction about MRGs in colorectal cancer. Besides, about the regulatory relationship of TFs on the prognosis-associated MRGs, we found that TFs (NR3C1, MYH11, MAF, and CBX7) positively regulated MRGs (ENPP2, PTGIS, GSTM5, and
P4HA3). Previous study indicates that the point mutation of MYH11 and the reduced expression quantity of CBX7 are related to the poor prognosis for CRC $[38,39]$. NR3C1 positively regulates the 4 prognosis-associated genes. Schlossmacher et al. indicated that glucocorticoid receptor encoded by NR3C1 promoted cell apoptosis through downregulating the expression of antiapoptotic proteins or inducing the expression of proapoptotic proteins [40]. However, there is no research on the regulatory role of NR3C1 in CRC, which may provide a new therapeutic target for metabolic treatment. Currently, targeted metabonomics analysis or nontargeted metabonomics analysis or the combination of both is employed to investigate the effect of differentially expressed metabolite or specific metabolite on the disease prognosis [41-43], among which, NMR spectroscopy is the representative of nontargeted metabonomics analysis method. Previously, Moolenaar et al. obtained the abnormally elevating N,N-dimethylglycine (DMG) induced by 


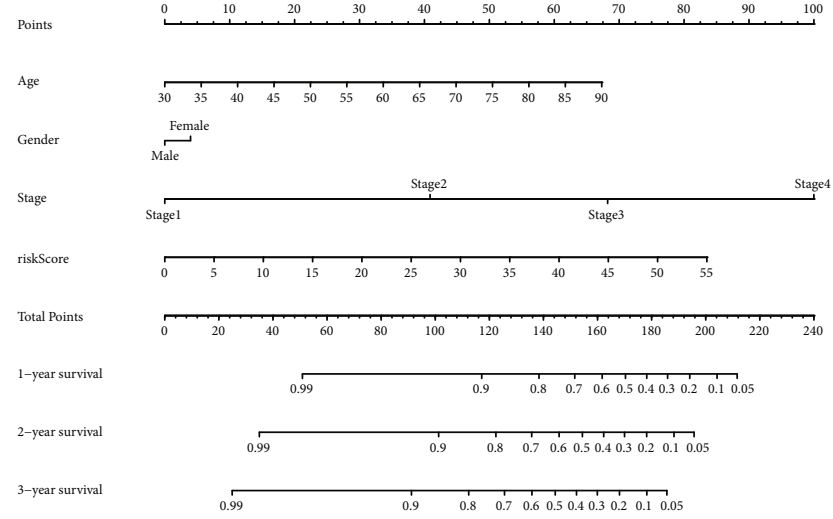

(a)

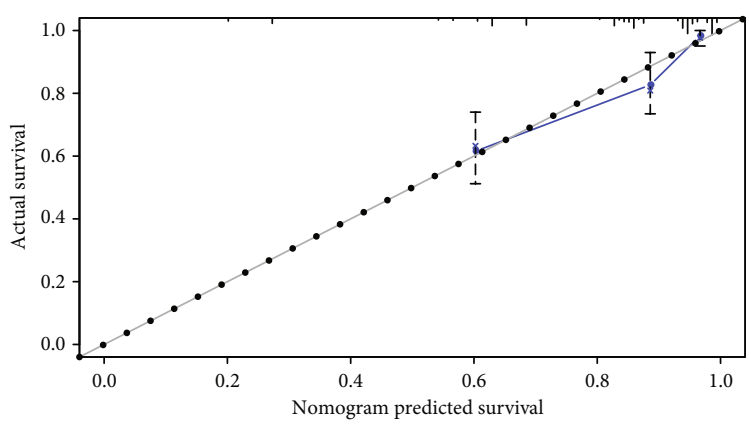

(c)
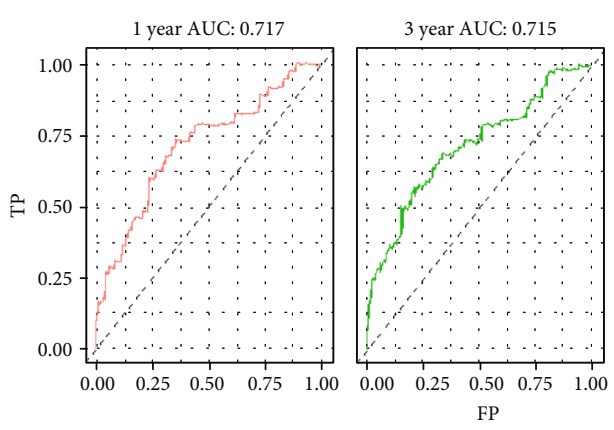

(e)

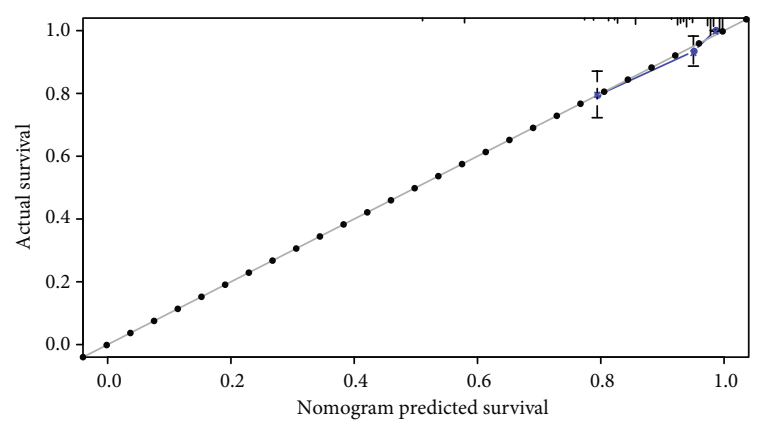

(b)

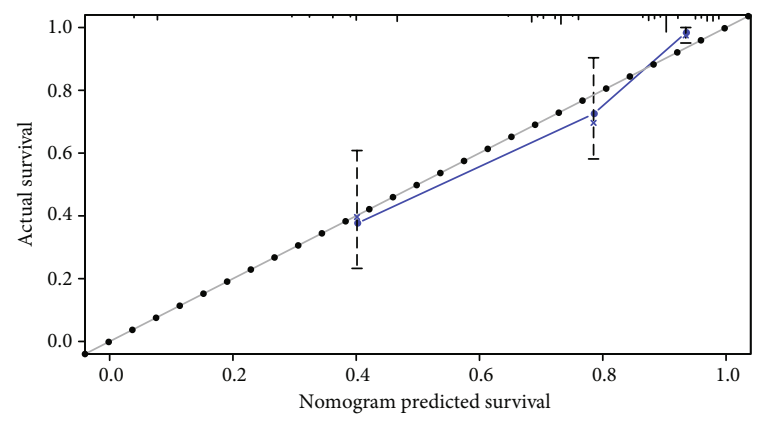

(d)

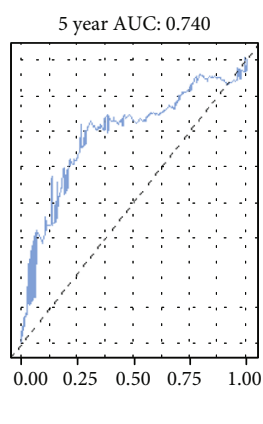

FIgURE 8: (a) The four-signature nomogram. (b-d) The 1-year, 3-year, and 5-year survival calibration curves. (e) The time-dependent ROC showed the comparison of AUCs among 1-year survival rate, 3-year survival rate, and 5-year survival rate based on the nomogram.

TABLE 6: The ten representative KEGG pathways in high- and low-risk groups.

\begin{tabular}{|c|c|c|c|c|c|}
\hline Names & Size & ES & NES & NOM $P$-value & FDR $q$-value \\
\hline \multicolumn{6}{|l|}{ High-risk group } \\
\hline KEGG_complement_and_coagulation_cascades & 69 & 0.684 & 2.100 & 0.002 & 0.030 \\
\hline KEGG_basal_cell_carcinoma & 55 & 0.620 & 2.077 & 0.002 & 0.022 \\
\hline KEGG_glycosaminoglycan_biosynthesis_chondroitin_sulfate & 22 & 0.795 & 2.048 & 0 & 0.021 \\
\hline KEGG_ECM_receptor_interaction & 84 & 0.708 & 2.021 & 0.006 & 0.022 \\
\hline KEGG_autoimmune_thyroid_disease & 50 & 0.724 & 2.018 & 0.002 & 0.018 \\
\hline \multicolumn{6}{|l|}{ Low-risk group } \\
\hline KEGG_propanoate_metabolism & 32 & -0.842 & -2.357 & 0 & 0 \\
\hline KEGG_peroxisome & 78 & -0.725 & -2.328 & 0 & 0 \\
\hline KEGG_fatty_acid_metabolism & 42 & -0.776 & -2.302 & 0 & $4.22 E-04$ \\
\hline KEGG_valine_leucine_and_isoleucine_degradation & 43 & -0.816 & -2.249 & 0 & $3.71 E-04$ \\
\hline KEGG_butanoate_metabolism & 34 & -0.764 & -2.147 & 0 & 0.003 \\
\hline
\end{tabular}




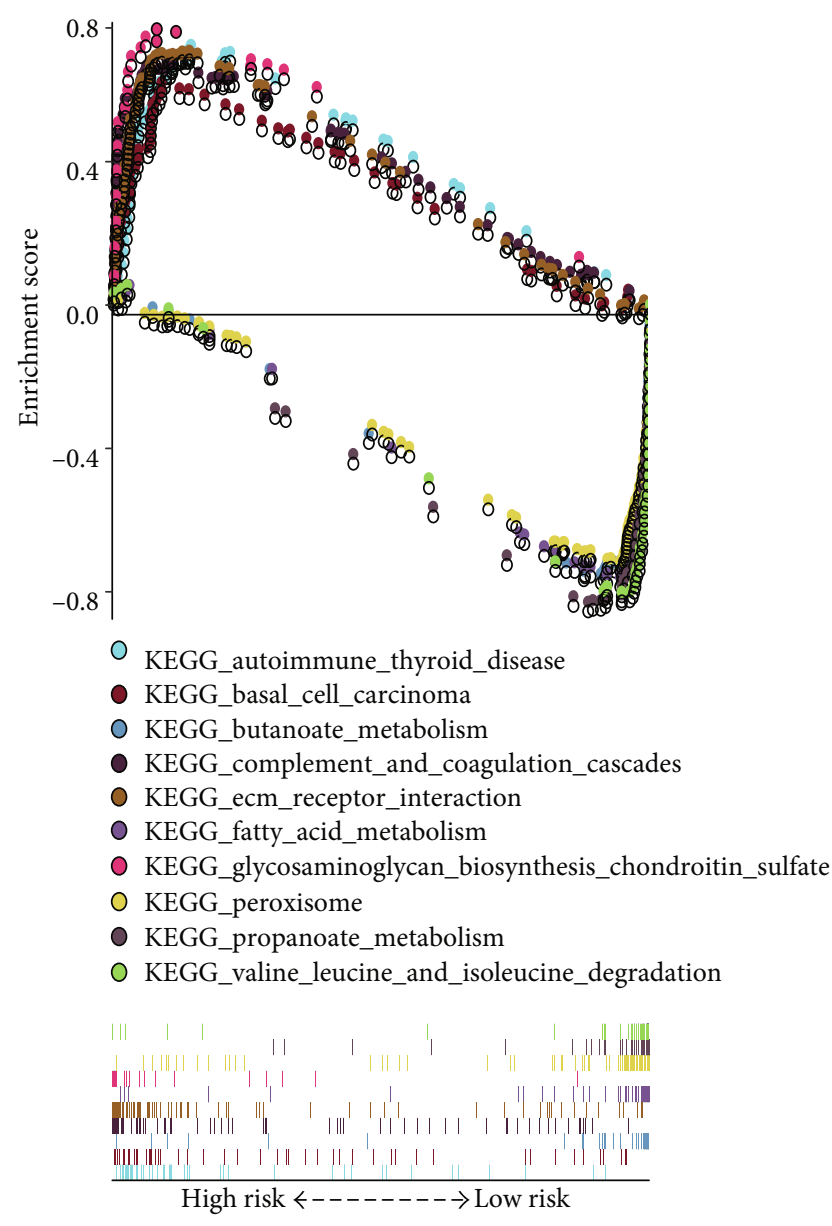

Figure 9: The representative ten enriched KEGG pathways in lowrisk and high-risk groups conducted from GSEA.

the congenital deficiency of enzyme dimethylglycine dehydrogenase (DMGDH) through 13C NMR spectroscopy and gas chromatography-mass spectrometry, which resulted in body odor [44]. In traditional metabonomics, the patient body fluid is collected to obtain the metabolic components to study the patient disease phenotype, but it is frequently dependent on the limited metabolic phenotype markers and is restricted by the quantities of sample metabolic components. Comparatively, our prognosis model utilized the high-throughput sequencing results to evaluate the DEGs and obtain their expression, and it was obtained based on the patient risk score acquired from the model algorithm, together with the patient clinical characteristics. Previously, the gene expression features are utilized to evaluate patient prognosis. O'Connell et al. constructed the multigene algorithms to quantify the prognosis for stage II/III CRC patients who received surgical treatment or combined with postoperative fluorouracil (FU) and leucovorin (LV) [45]. Agesen combined patients, populations, and Affymetrix exon-level microarrays to display a 13 gene-based classifier for predicting the prognosis of stage II CRC through COX regression analysis [46]. These studies have quantified the prognosis evaluation at the molecular level. Additionally, this MRGbased prognosis model expanded the gene biological functions. We conducted GSEA on high-risk group and low- risk group, finding numerous KEGG enriched pathways, most of which were related to metabolism. A vast majority of these pathways were enriched in the low-risk group, including propanoate metabolism pathway and fatty acid metabolism pathway. In recent years, research on the influence of lipid metabolic pathway on CRC development has always been a hotspot. Kazlauskas suggested that LPA played an important role in stimulating tumor angiogenesis [47], thus regulating tumor metastasis, while angiogenesis in tumor tissues usually promotes tumor growth and metastasis [48]. Yeh et al. employed the microarray-bioinformatics analysis methods to reveal that the activation of fatty acid pathway promoted CRC genesis and development at gene level [49]. Recently, Wang et al. discovered that the activation of the CPT1A-mediated fatty acid oxidation pathways suppressed anoikis to accelerate CRC development and metastasis [50]. And for propanoate metabolism pathway, Perroud et al. illustrated that 31 proteins in the propanoate metabolism pathway were associated with the genesis of clear cell RCC (ccRCC) [51]. However, how the propanoate metabolism pathway affects the genesis and development of colorectal cancer is still being probed. Moreover, compared with the high-risk group, it was feasible to apply the metabolic therapy in the low-risk CRC group. Such a result indirectly verified the feasibility to treat early CRC with metabolic-targeted therapy.

\section{Conclusions}

In conclusion, we constructed a prognostic model based on six MRGs to predict the prognosis of CRC by using the bioinformatics method. Univariate and multivariate Cox regression analysis for the training group, validation group, and overall samples verified the prognostic value of the prognostic model. Moreover, the TF-MRG network and PPI network revealed novel molecular regulatory targets about metabolism in CRC. GSEA for biological functions based on the prognostic model not only provided fresh sights about the therapeutic target but also facilitated the individualized treatment for CRC patients.

\section{Data Availability}

We analyzed the publicly available datasets in this study. All data were extracted from TCGA, MSigDB, and Cistrome Project.

\section{Consent}

Consent for participation from all patients was obtained through The Cancer Genome Atlas Project.

\section{Conflicts of Interest}

The authors declared that they had no conflict of interests.

\section{Authors' Contributions}

$\mathrm{Xu}$ YC and Sun YL designed the study, Zhang Y carried out the data collection, Guo YC and Yang $\mathrm{ZH}$ conducted the data 
analysis, Sun YL drafted the manuscript, and Xu YC revised the manuscript. All authors read and approved the final manuscript.

\section{Supplementary Materials}

Table S1: the 6475 DEGs. Table S2: the 289 differentially expressed MRGs. Table S3: the 72 differentially expressed TFs. (Supplementary materials)

\section{References}

[1] F. Bray, J. Ferlay, I. Soerjomataram, R. L. Siegel, L. A. Torre, and A. Jemal, "Global cancer statistics 2018: GLOBOCAN estimates of incidence and mortality worldwide for 36 cancers in 185 countries," CA: a Cancer Journal for Clinicians, vol. 68, no. 6, pp. 394-424, 2018.

[2] E. H. Schreuders, A. Ruco, L. Rabeneck et al., "Colorectal cancer screening: a global overview of existing programmes," Gut, vol. 64, no. 10, pp. 1637-1649, 2015.

[3] S. Yachida, S. Mizutani, H. Shiroma et al., "Metagenomic and metabolomic analyses reveal distinct stage-specific phenotypes of the gut microbiota in colorectal cancer," Nature Medicine, vol. 25, no. 6, pp. 968-976, 2019.

[4] S. Jones, W.-D. Chen, G. Parmigiani et al., "Comparative lesion sequencing provides insights into tumor evolution," Proceedings of the National Academy of Sciences, vol. 105, no. 11, pp. 4283-4288, 2008.

[5] Z. Zhai, X. Yu, B. Yang et al., "Colorectal cancer heterogeneity and targeted therapy: clinical implications, challenges and solutions for treatment resistance," Seminars in Cell \& Developmental Biology, vol. 64, pp. 107-115, 2017.

[6] J. L. Westra, M. Schaapveld, H. Hollema et al., "Determination of TP53 mutation is more relevant than microsatellite instability status for the prediction of disease-free survival in adjuvant-treated stage III colon cancer patients," Journal of Clinical Oncology, vol. 23, no. 24, pp. 5635-5643, 2005.

[7] W. Weng, N. Liu, Y. Toiyama et al., "Novel evidence for a PIWI-interacting RNA (piRNA) as an oncogenic mediator of disease progression, and a potential prognostic biomarker in colorectal cancer," Molecular Cancer, vol. 17, no. 1, p. 16, 2018.

[8] N. Alikhani, R. D. Ferguson, R. Novosyadlyy et al., "Mammary tumor growth and pulmonary metastasis are enhanced in a hyperlipidemic mouse model," Oncogene, vol. 32, no. 8, pp. 961-967, 2013.

[9] X. Shu, Y. B. Xiang, N. Rothman et al., "Prospective study of blood metabolites associated with colorectal cancer risk," International Journal of Cancer, vol. 143, no. 3, pp. 527-534, 2018.

[10] J. Chen, J. Xie, Z. Jiang, B. Wang, Y. Wang, and X. Hu, "Shikonin and its analogs inhibit cancer cell glycolysis by targeting tumor pyruvate kinase-M2," Oncogene, vol. 30, no. 42, pp. 4297-4306, 2011.

[11] S. Mei, C. A. Meyer, R. Zheng et al., "Cistrome cancer: a web resource for integrative gene regulation modeling in cancer," Cancer Research, vol. 77, no. 21, pp. e19-e22, 2017.

[12] P. Shannon, A. Markiel, O. Ozier et al., "Cytoscape: a software environment for integrated models of biomolecular interaction networks," Genome Research, vol. 13, no. 11, pp. 24982504, 2003.
[13] R. Tibshirani, "The lasso method for variable selection in the Cox model," Statistics in Medicine, vol. 16, no. 4, pp. 385395, 1997.

[14] A. Subramanian, P. Tamayo, V. K. Mootha et al., "Gene set enrichment analysis: a knowledge-based approach for interpreting genome-wide expression profiles," Proceedings of the National Academy of Sciences of the United States of America, vol. 102, no. 43, pp. 15545-15550, 2005.

[15] S. Kaitaniemi, H. Elovaara, K. Grön et al., "The unique substrate specificity of human AOC2, a semicarbazide-sensitive amine oxidase," Cellular and Molecular Life Sciences, vol. 66, no. 16, pp. 2743-2757, 2009.

[16] E. Shoshan, R. R. Braeuer, T. Kamiya et al., "NFAT1 directly regulates IL8 and MMP3 to promote melanoma tumor growth and metastasis," Cancer Research, vol. 76, no. 11, pp. 31453155, 2016.

[17] Y. Lu, W. Lemon, P. Y. Liu et al., "A gene expression signature predicts survival of patients with stage I non-small cell lung cancer," PLoS Medicine, vol. 3, no. 12, article e467, 2006.

[18] Z. Zhao, Y. Xiao, P. Elson et al., "Plasma lysophosphatidylcholine levels: potential biomarkers for colorectal cancer," Journal of Clinical Oncology, vol. 25, no. 19, pp. 2696-2701, 2007.

[19] D. Zanini, L. H. Manfredi, L. P. Pelinson et al., "ADA activity is decreased in lymphocytes from patients with advanced stage of lung cancer," Medical Oncology, vol. 36, no. 9, 2019.

[20] T. J. Kelly, A. L. Souza, C. B. Clish, and P. Puigserver, "A hypoxia-induced positive feedback loop promotes hypoxiainducible factor 1alpha stability through miR-210 suppression of glycerol-3-phosphate dehydrogenase 1-like," Molecular and Cellular Biology, vol. 31, no. 13, pp. 2696-2706, 2011.

[21] V. K. Hill, C. Ricketts, I. Bieche et al., "Genome-wide DNA methylation profiling of $\mathrm{CpG}$ islands in breast cancer identifies novel genes associated with tumorigenicity," Cancer Research, vol. 71, no. 8, pp. 2988-2999, 2011.

[22] N. Fujiwara, H. Nakagawa, K. Enooku et al., "CPT2 downregulation adapts HCC to lipid-rich environment and promotes carcinogenesis via acylcarnitine accumulation in obesity," Gut, vol. 67, no. 8, pp. 1493-1504, 2018.

[23] L. Zhou, Y. Du, L. Kong, X. Zhang, and Q. Chen, "Identification of molecular target genes and key pathways in hepatocellular carcinoma by bioinformatics analysis," OncoTargets and therapy., vol. Volume 11, pp. 1861-1869, 2018.

[24] H. Xiao, P. Chen, G. Zeng, D. Xu, X. Wang, and X. Zhang, "Three novel hub genes and their clinical significance in clear cell renal cell carcinoma," Journal of Cancer, vol. 10, no. 27, pp. 6779-6791, 2019.

[25] B. W. Wong, X. Wang, A. Zecchin et al., "The role of fatty acid $\beta$-oxidation in lymphangiogenesis," Nature, vol. 542, no. 7639, pp. 49-54, 2017.

[26] T. Wang, J. F. Fahrmann, H. Lee et al., "JAK/STAT3-regulated fatty acid $\beta$-oxidation is critical for breast cancer stem cell selfrenewal and chemoresistance," Cell Metabolism, vol. 27, no. 6, p. 1357, 2018.

[27] N. Xu, Y.-P. Wu, Z.-B. Ke et al., "Identification of key DNA methylation-driven genes in prostate adenocarcinoma: an integrative analysis of TCGA methylation data," Journal of Translational Medicine, vol. 17, no. 1, p. 311, 2019.

[28] B. Griffiths, C. A. Lewis, K. Bensaad et al., "Sterol regulatory element binding protein-dependent regulation of lipid synthesis supports cell survival and tumor growth," Cancer Metab., vol. 1, no. 1, p. 3, 2013. 
[29] K. Zaugg, Y. Yao, P. T. Reilly et al., "Carnitine palmitoyltransferase 1C promotes cell survival and tumor growth under conditions of metabolic stress," Genes \& Development, vol. 25, no. 10, pp. 1041-1051, 2011.

[30] J. M. Tirado-Vélez, I. Joumady, A. Sáez-Benito, I. Cózar-Castellano, and G. Perdomo, "Inhibition of fatty acid metabolism reduces human myeloma cells proliferation," PLoS One, vol. 7, no. 9, article e46484, 2012.

[31] J. Li, Q. Huang, X. Long et al., "CD147 reprograms fatty acid metabolism in hepatocellular carcinoma cells through Akt $/ \mathrm{m}$ TOR/SREBP1c and P38/PPAR $\alpha$ pathways," Journal of Hepatology, vol. 63, no. 6, pp. 1378-1389, 2015.

[32] H. Ruan, Z. Song, Q. Cao et al., "IMPDH1/YB-1 positive feedback loop assembles cytoophidia and represents a therapeutic target in metastatic tumors," Molecular Therapy, vol. 28, no. 5, pp. 1299-1313, 2020.

[33] Y. An, H. Cai, Y. Yang et al., "Identification of ENTPD8 and cytidine in pancreatic cancer by metabolomic and transcriptomic conjoint analysis," Cancer Science, vol. 109, no. 9, pp. 2811-2821, 2018.

[34] M. F. Orth, J. S. Gerke, T. Knösel et al., "Functional genomics identifies AMPD2 as a new prognostic marker for undifferentiated pleomorphic sarcoma," International journal of cancer., vol. 144, no. 4, pp. 859-867, 2019.

[35] J. A. Wawrzyniak, A. Bianchi-Smiraglia, W. Bshara et al., "A purine nucleotide biosynthesis enzyme guanosine monophosphate reductase is a suppressor of melanoma invasion," Cell Reports, vol. 5, no. 2, pp. 493-507, 2013.

[36] L. Feldbrügge, A. C. Moss, E. U. Yee et al., "Expression of ectonucleoside triphosphate diphosphohydrolases-2 and -3 in the enteric nervous system affects inflammation in experimental colitis and Crohn's disease," Journal of Crohn's \& Colitis, vol. 11, no. 9, pp. 1113-1123, 2017.

[37] X. Luo, H. Yu, Y. Song, and T. Sun, "Integration of metabolomic and transcriptomic data reveals metabolic pathway alteration in breast cancer and impact of related signature on survival," Journal of Cellular Physiology, vol. 234, no. 8, pp. 13021-13031, 2018.

[38] S. Roychowdhury, M. K. Iyer, D. R. Robinson et al., "Personalized oncology through integrative high-throughput sequencing: a pilot study," Science translational medicine, vol. 3, no. 111, article 111ra121, 2011.

[39] P. Pallante, L. Terracciano, V. Carafa et al., "The loss of the CBX7 gene expression represents an adverse prognostic marker for survival of colon carcinoma patients," European Journal of Cancer, vol. 46, no. 12, pp. 2304-2313, 2010.

[40] G. Schlossmacher, A. Stevens, and A. White, "Glucocorticoid receptor-mediated apoptosis: mechanisms of resistance in cancer cells," The Journal of Endocrinology, vol. 211, no. 1, pp. 17-25, 2011.

[41] H. Zha, Y. Cai, Y. Yin, Z. Wang, K. Li, and Z.-J. Zhu, "SWATHtoMRM: development of high-coverage targeted metabolomics method using SWATH technology for biomarker discovery," Analytical Chemistry, vol. 90, no. 6, pp. 4062-4070, 2018.

[42] Y. Chen, Z. Zhou, W. Yang et al., "Development of a dataindependent targeted metabolomics method for relative quantification using liquid chromatography coupled with tandem mass spectrometry," Analytical Chemistry, vol. 89, no. 13, pp. 6954-6962, 2017.
[43] X. Wang, D. Wang, Y. Wang, P. Zhang, Z. Zhou, and W. Zhu, "A combined non-targeted and targeted metabolomics approach to study the stereoselective metabolism of benalaxyl enantiomers in mouse hepatic microsomes," Environmental Pollution, vol. 212, pp. 358-365, 2016.

[44] S. H. Moolenaar, J. Poggi-Bach, U. F. H. Engelke et al., "Defect in dimethylglycine dehydrogenase, a new inborn error of metabolism: NMR spectroscopy study," Clinical Chemistry, vol. 45, no. 4, pp. 459-464, 1999.

[45] M. J. O'Connell, I. Lavery, G. Yothers et al., "Relationship between tumor gene expression and recurrence in four independent studies of patients with stage II/III colon cancer treated with surgery alone or surgery plus adjuvant fluorouracil plus leucovorin," Journal of Clinical Oncology, vol. 28, no. 25, pp. 3937-3944, 2010.

[46] T. H. Ågesen, A. Sveen, M. A. Merok et al., "ColoGuideEx: a robust gene classifier specific for stage II colorectal cancer prognosis," Gut, vol. 61, no. 11, pp. 1560-1567, 2012.

[47] A. Kazlauskas, "Lysophosphatidic acid contributes to angiogenic homeostasis," Experimental Cell Research, vol. 333, no. 2, pp. 166-170, 2015.

[48] J. Folkman, "Role of angiogenesis in tumor growth and metastasis," Seminars in Oncology, vol. 29, no. 6Q, pp. 15-18, 2002.

[49] C.-S. Yeh, J.-Y. Wang, T.-L. Cheng, C.-H. Juan, C.-H. Wu, and S.-R. Lin, "Fatty acid metabolism pathway play an important role in carcinogenesis of human colorectal cancers by microarray-bioinformatics analysis," Cancer letters., vol. 233, no. 2, pp. 297-308, 2006.

[50] Y. N. Wang, Z. L. Zeng, J. Lu et al., "CPT1A-mediated fatty acid oxidation promotes colorectal cancer cell metastasis by inhibiting anoikis," Oncogene, vol. 37, no. 46, pp. 6025-6040, 2018.

[51] B. Perroud, J. Lee, N. Valkova et al., "Pathway analysis of kidney cancer using proteomics and metabolic profiling," Molecular Cancer, vol. 5, no. 1, p. 64, 2006. 